\title{
Hedonic price equilibria, stable matching, and optimal transport: equivalence,topology, and uniqueness
}

\section{Pierre-Andrè Chiappori Robert J. McCann Lars Nesheim}

The Institute for Fiscal Studies Department of Economics, UCL cemmap working paper CWP24/07 


\title{
Hedonic price equilibria, stable matching, and optimal transport: equivalence, topology, and uniqueness $^{1}$
}

\author{
Pierre-André Chiappori ${ }^{2}$, Robert J. McCann ${ }^{3}$, and Lars Nesheim ${ }^{4}$
}

September 28, 2007

\begin{abstract}
${ }^{1}$ It is a pleasure to thank Ivar Ekeland, whose conferences at the Banff International Research Station (BIRS) in 2004 and 2005 brought us together, and whose work on hedonic pricing [12], [13] inspired our investigation. RJM also wishes to thank Najma Ahmad, Wilfrid Gangbo, and Hwakil Kim, for fruitful conversations related to the subtwist criterion for uniqueness and the resolution of Birkhoff's problem, Nathan Killoran for his help with the references, and Nassif Ghoussoub for insightful remarks. The authors are pleased to acknowledge the support of Natural Sciences and Engineering Research Council of Canada Grant 217006-03 and United States National Science Foundation Grants 0241858, 0433990, 0532398 and DMS-0354729. They also acknowledge support through CEMMAP from the Leverhulme Trust and the UK Economic and Social Research Council grant RES-589-28-0001. (c)2007 by the authors.

${ }^{2}$ Department of Economics, Columbia University, USA, pc2167@columbia.edu

${ }^{3}$ Department of Mathematics, University of Toronto, Canada, mccann@math. toronto. edu

${ }^{4}$ Centre for Microdata Methods and Practice (CEMMAP), University College London and the Institute for Fiscal Studies, UK, 1.nesheim@ucl.ac.uk
\end{abstract}




\begin{abstract}
Hedonic pricing with quasilinear preferences is shown to be equivalent to stable matching with transferable utilities and a participation constraint, and to an optimal transportation (Monge-Kantorovich) linear programming problem. Optimal assignments in the latter correspond to stable matchings, and to hedonic equilibria. These assignments are shown to exist in great generality; their marginal indirect payoffs with respect to agent type are shown to be unique whenever direct payoffs vary smoothly with type. Under a generalized Spence-Mirrlees condition the assignments are shown to be unique and to be pure, meaning the matching is one-to-one outside a negligible set. For smooth problems set on compact, connected type spaces such as the circle, there is a topological obstruction to purity, but we give a weaker condition still guaranteeing uniqueness of the stable match. An appendix resolves an old problem (\# 111) of Birkhoff in probability and statistics [5], by giving a necessary and sufficient condition on the support of a joint probability to guarantee extremality among all joint measures with the same marginals.
\end{abstract}




\section{Introduction}

The goal of this note is to establish and exploit a general, structural equivalence result between three families of models, two of which are familiar to economists while the third belongs in mathematics and operational research. Specifically, we consider a general framework for studying hedonic price problems with quasilinear preferences, and show that it is equivalent to a matching model with transferable utilities. From a mathematical perspective, both problems can in turn be rephrased under the form of a linear program, in fact an optimal transportation problem of Monge-Kantorovich type. Secondly, we argue that, due to the wide body of knowledge about linear programming in general, and optimal transportation in particular (see for example [2] and [39]), the reduction of the model to this form seems not only conceptually clearer, but better adapted to bringing powerful methods of theoretical and computational analysis to bear on the question.

As an illustration, we first provide a general existence result for the models under consideration. The result is valid for matching as well as hedonic pricing models. It applies to multi-dimensional problems, and does not require single crossing conditions à la Spence-Mirrlees. ${ }^{1}$ In the smooth setting, we establish uniqueness of the marginal payoff with respect to type, even though the optimal matching can be non-unique.

We also clarify the role of the well known Spence-Mirrlees condition. In the one-dimensional setting usually considered by economists, the condition guarantees some form of assortative matching - which, in turn, implies that the equilibrium is both unique and pure, (purity meaning the matching is one-to-one for almost all agents). As we discuss below, the notions of purity and uniqueness generalize naturally to multi-dimensions, whereas the notion of assortative matching does not.

We first describe a generalization of the Spence-Mirrlees condition that is valid in general type spaces, does not require differentiability of the surplus function, allows for non-participation, and is not dependent on the coordinates (i.e. the parametrization) of the problem. We then show that this condition, while sufficient, is not necessary for uniqueness of the stable match. In particular, we provide an example in which the stable match is unique although the Spence-Mirrlees condition is violated. In such a case, however, the solution fails to be pure. That is, when Spence-Mirrlees does not hold, it may be the case that identical agents on one side of the market are matched with different counterparts, a situation that might be interpreted in terms of mixed strategies. Lastly, we provide a new and weaker condition that guarantees uniqueness of the stable match in the matching model (or of the equilibrium in the hedonic model) even in the absence of pure matching.

In both hedonic models and matching (or assignment) models, much of the intuition economists have developed is restricted to models in which either there

\footnotetext{
${ }^{1}$ Our approach can be viewed as a simplification of the more complex (but ultimately equivalent) formulation of the problem as a convex nonlinear program due to Ekeland [12], [13], and subsequently developed in his joint work with Carlier [9].
} 
is a finite number of types or in which the agents in the model can be described by a one dimensional characteristic under a single-crossing property. Much of the discussion in the theoretical and empirical literature focuses on whether there is positive assortative matching. The optimal transportation approach, initially introduced by Shapley and Shubik [35] and extended to a continuous setting by Gretsky, Ostroy and Zame [18], opens up the study of hedonic and matching models with multidimensional characteristics, general surplus functions, and general distributions of types. This paper reviews the relevant results from this literature showing how they can be applied in these economic settings. Further, it highlights some significant issues related to the geometry and topology of the type spaces which have not previously been explored, neither in the economics nor the mathematics literature. For example, when agent types are located on a circle, a sphere, or products thereof (such as a periodic square), no smooth generalization of the Spence-Mirrlees condition can hold, and stable matchings (or assignments) are not generally pure. The subtwist criterion we introduce resolves assignment uniqueness in some of these settings, but leaves others as open challenges. An interesting question that we do not discuss relates to the continuity or smoothness of the dependence of the buyer's characteristics on those of the seller with whom he chooses to match. Significant recent progress on this question is surveyed by Villani in [41].

Finally, in an appendix we resolve a long-standing question in probability and statistics which can be traced back to Birkhoff [5]: we give a necessary and sufficient condition on the support of a joint probability measure to guarantee its extremality among measures which share its marginals. We then exploit this criterion to establish our unique assignment results.

Our work builds upon and extends several existing contributions in economics and in mathematics. Gretsky, Ostroy and Zame [18], [19] also study the matching of buyers and sellers in an economy with potentially a continuum of agents. In their economy, buyers and sellers who match are not free to trade any contract. Rather each seller is endowed with a single contract that they can sell or not. Their economy is thus a hedonic endowment economy, while ours can be seen as hedonic production one. Gretsky, Ostroy and Zame [18] shows that equilibrium in the endowment economy is equivalent to an optimal transportation problem and to a matching problem. They also prove that equilibrium exists. Gretsky, Ostroy and Zame [19] analyze the equilibrium in the endowment economy and focus on its links with perfect competition. They prove that in the continuum economy, perfect competition (the inability of individuals to influence price) obtains when the social gains function (i.e. the value of the primal program) is differentiable, or equivalently when the solution to the dual is unique. However, they do not give conditions on the exogenous data which guarantee this uniqueness; nor do they analyze uniqueness of the optimal assignment or purity of the solution.

Ekeland [12], [13] also studies the hedonic model presented here. He shows that the model can be reformulated as a convex nonlinear program. He proves existence of equilibrium under conditions very similar to ours and uniqueness and purity under an analogous version of the multidimensional Spence-Mirrlees 
condition. He concludes that computation of the equilibrium in the general case is difficult and remains and unsolved problem.

On the mathematical side, multi-dimensional generalizations of the SpenceMirrlees condition developed through work of many authors, including Brenier, Caffarelli, Gangbo, McCann, Carlier, Ambrosio, Rigot, Ma, Trudinger, Wang, Bernard, Buffoni, Bertrand, Agrachev and Lee, as surveyed by Villani [39] [41]. Special cases of costs satisfying the subtwist condition were investigated by Uckelmann [37], McCann [29], Gangbo and McCann [17], Plakhov [32], and Ahmad [1]. Relevant contributions to Birkhoff's problem were made by Douglas [10], Lindenstrauss [26], Beneš and Stěpán [3] and Hestir and Williams [21], among others.

\section{The basic framework}

\subsection{The competitive hedonic model}

Consider a competitive spot market in which sellers produce and buyers acquire objects or contracts $z$ which come in a wide range of qualities $z \in Z_{0}$. What is peculiar to many competitive hedonic markets, including those for housing, workers, vegetables, automobiles, pensions, insurance contracts, and many others, is that in the spot market for these contracts, a large number of buyers and sellers trade fixed quantities, often small, of contracts whose value (to buyers and/or sellers) depend on quantifiable qualities, or characteristics. ${ }^{2}$ These "hedonic" characteristics are known to the buyers and/or sellers at the time of the transaction and as a result are reflected in the equilibrium market price.

Assuming buyer and seller preferences have been specified, the problem posed by such a market is to decide how supply equilibrates with demand to determine the set of contracts actually exchanged on the market (or the set of commodities actually produced and consumed), and the price $P(z)$ at which each type of contract is traded. Note that such an equilibrium implicitly defines a pairing or matching of buyers with sellers who choose to enter into this market by agreeing to contract or exchange with each other.

Standing hypotheses: The sets $X_{0}, Y_{0}, Z_{0}$, of buyer, seller, and contract types, may be modeled Borel subsets of complete separable metric spaces, possibly multidimensional. To allow for the possibility that some agents choose not to participate, we augment the spaces $X:=X_{0} \cup\left\{\emptyset_{X}\right\}, Y:=Y_{0} \cup\left\{\emptyset_{Y}\right\}$ and $Z=Z_{0} \cup\left\{\emptyset_{Z}\right\}$ by including an isolated point in each: a partner $\emptyset_{X}$ for any unmatched sellers, a partner $\emptyset_{Y}$ for any unmatched buyers, and the null contract $\emptyset_{Z}$. Preferences are encoded into functions representing the utility $u(x, z)$ of product $z \in Z$ to buyer $x \in X$, and the utility (disutility or cost) $v(y, z)$ of product $z \in Z$ to seller $y \in Y$. These utility functions $u: X \times Z \longrightarrow \mathbf{R} \cup\{-\infty\}$ and $v: Y \times Z \longrightarrow \cup\{+\infty\}$ are specified a priori, along with non-negative Borel measures $\mu_{0}$ on $X_{0}$ and $\nu_{0}$ on $Y_{0}$ of finite total mass representing the distribu-

\footnotetext{
${ }^{2}$ These models also apply to markets where prices are nonlinear in quantities because different quantities are not perfect substitutes and cannot be freely traded.
} 
tion of buyer and seller types throughout the population. The utility functions are constrained so that neither the dummy buyer type $\emptyset_{X}$ nor the dummy seller type $\emptyset_{Y}$ can participate in any exchange save the null contract:

$$
u\left(\emptyset_{X}, z\right)=\left\{\begin{array}{cl}
0 & \text { if } z=\emptyset_{Z}, \quad v\left(\emptyset_{Y}, z\right)=\left\{\begin{array}{cl}
0 & \text { if } z=\emptyset_{Z}, \\
-\infty & \text { else; }
\end{array}\right. \text { else }
\end{array}\right.
$$

while the measures $\mu_{0}$ and $\nu_{0}$ are extended to $X$ and $Y$ by assigning mass $\nu_{0}\left(Y_{0}\right)+1$ and $\mu_{0}\left(X_{0}\right)+1$ to the points $\emptyset_{X}$ and $\emptyset_{Y}$ respectively:

$$
\mu:=\mu_{0}+\left(\nu_{0}\left(Y_{0}\right)+1\right) \delta_{\emptyset_{X}} \quad \nu:=\nu_{0}+\left(\mu_{0}\left(X_{0}\right)+1\right) \delta_{\emptyset_{Y}} .
$$

The augmented measures balance $\mu[X]=\nu[Y]<\infty$, so we can renormalize them to be probability measures (i.e. have unit mass) without loss of generality. ${ }^{3}$

To guarantee the convergence of various integrals, and attainment of various suprema and infima, we assume throughout (and tacitly hereafter) that $u(x, z)>-\infty$ extends upper semicontinuously to the completion of $X \times Z$ and $v(y, z)<\infty$ lower semicontinuously to the completion of $Y \times Z$. We normalize the utility of the null-contract to be zero

$$
u\left(x, \emptyset_{Z}\right)=0=v\left(y, \emptyset_{Z}\right),
$$

which can be achieved without loss of generality if the reserve utilities $u\left(x, \emptyset_{Z}\right) \in$ $L^{1}(X, d \mu)$ and $v\left(y, \emptyset_{Z}\right) \in L^{1}(Y, d \nu)$ are continuous and integrable, by subtracting them from $u$ and $v$.

Define the pairwise surplus function

$$
s(x, y)=\sup _{z \in Z} u(x, z)-v(y, z) .
$$

We assume that for each pair the supremum is attained. Further, in case $u$ or $v$ is discontinuous or $Z$ fails to be compact [22], we assume the set of contracts

$$
Z(x, y)=\arg \max _{z \in Z} u(x, z)-v(y, z)
$$

that maximize the surplus (4) is non-empty, compact, and depends upper hemicontinuously on $(x, y) \in X \times Y$. It is well-known [22] that there exists a measurable selection, i.e., a Borel function $z_{0}: X \times Y \rightarrow Z$ such that $z_{0}(x, y)$ is contained in $Z(x, y)$ for all $(x, y)$.

In case $u$ or $-v$ fails to be bounded, we assume there exist lower semicontinuous real-valued functions $\bar{q} \in L^{1}(X, d \mu)$ and $\bar{r} \in L^{1}(Y, d \nu)$ such that

$$
\sup _{x \in X} u(x, z)-\bar{q}(x) \leq \inf _{y \in Y} v(y, z)+\bar{r}(y)
$$

for all $z \in Z$. This is roughly equivalent to the existence of prices on $Z$ which make the indirect utilities integrable. Given any Borel map $f: \operatorname{Dom} f \longrightarrow Z$

\footnotetext{
${ }^{3}$ The excessive mass on $\delta_{\emptyset_{X}}$ and $\delta_{\emptyset_{Y}}$ ensures that at least some null types match with each other and obtain a pairwise surplus of zero.
} 
on a subset $\operatorname{Dom} f \subset X$, we define a measure on $Z$, called the push-forward of $\mu$ through $f$, by the formula $\left(f_{\#}\right) \mu(B)=\mu\left(f^{-1}(B)\right)$ for Borel $B \subset Z$.

Suppose $P: Z \longrightarrow \mathbf{R} \cup\{ \pm \infty\}$ denotes the competitive market price of quality $z \in Z$. To allow non-participation, it is subject to the constraint $P\left(\emptyset_{Z}\right)=0$. We assume that buyer utility is linear in price so that in such a market, the indirect utility available to buyer type $x \in X$ is defined by the quasi-linear utility maximization

$$
U(x)=\sup _{z \in Z}\{u(x, z)-P(z)\} .
$$

Here $U(x) \geq 0$ is non-negative since $\emptyset_{Z} \in Z$; each buyer $x$ retains the right not to consume. Similarly, we assume seller utility is linear in price so that the indirect utility available to seller type $y \in Y$ is given by the utility maximization

$$
V(y)=\sup _{z \in Z}\{P(z)-v(y, z)\},
$$

with $V(y) \geq 0$ and vanishing in the case of non-participation. We make the conventions $(-\infty)-(-\infty)=-\infty$ and $\infty-\infty=-\infty$ to resolve ambiguities in (7)-(8).

Let $\alpha$ be a non-negative measure on $X \times Y \times Z$. The support of $\alpha$ refers to the smallest closed set $\operatorname{Spt}(\alpha) \subseteq X \times Y \times Z$ of full mass. The measure $\alpha$ represents an assignment of buyers and sellers to each other and to products. We use the push-forward notation to denote its marginal projections $\pi_{\#}^{X} \alpha$ and $\pi_{\#}^{Y} \alpha$ under mappings such as $\pi^{X}(x, y, z)=x$ and $\pi^{Y}(x, y, z)=y$ on $X \times Y \times Z$.

The pair $(\alpha, P)$ is an hedonic equilibrium if these projections coincide with the initial measures on each set:

$$
\begin{aligned}
& \pi_{\#}^{X} \alpha=\mu \\
& \pi_{\#}^{Y} \alpha=\nu
\end{aligned}
$$

and if, for $\alpha$-almost all points $(x, y, z) \in \operatorname{Spt} \alpha$, we have that

$$
\begin{aligned}
& U(x)=u(x, z)-P(z) \\
& V(y)=P(z)-v(y, z) .
\end{aligned}
$$

In such an equilibrium, each triple $(x, y, z) \in \operatorname{Spt} \alpha$ represents a mutually agreeable exchange of contract $z$ between seller $y$ and buyer $x$, where $z$ is a contract most favored by both seller $y$ and buyer $x$ independently, given market prices $P$. The prices are market clearing, in the sense that the assignment $\alpha$ is consistent with the utility maximization of both buyers and sellers (10) while simultaneously balancing supply with demand (9). Since the prices of untraded commodities potentially affect the indirect utilities $U(x)$ and $V(y)$, prices for these commodities are subject to upper and lower bounds in a market at equilibrium. We use the term market clearing pair synonymously with equilibrium pair. This notion of equilibrium allows for the possibility that some agents are indifferent between multiple qualities in $Z$. Indeed, when $\alpha$ assigns a buyer $x$ 
to multiple sellers or contracts, we may interpret the conditional distribution implied by $\alpha$ as a mixed strategy for buyer $x$. In such an equilibrium, the assignment $\alpha$ must still ensure that the number of buyers and sellers of each contract type are compatible in the sense of $(9)$.

\subsection{The associated matching problem}

Similarly, models of one-to-one matching with transferable utility are used to analyze marriage markets, labour markets and the matching of students to schools to understand who matches with whom in an equilibrium stable matching. In these models the partners on each side of the matching have characteristics that affect the surplus that may be attained by any matched pair. The characteristics of the agents matched are reflected in the equilibrium matching and in the utility payoffs that each agent obtains.

As stated in introduction, there is a natural, one-to-one correspondence between hedonic models and matching problems. We first characterize the pairwise matching problem derived from the hedonic price model just described.

Characterization: The basic idea is very simple. For each pair $(x, y) \in$ $X \times Y$, recall the pairwise surplus function $s$ defined in (4). In words, whenever a buyer $x$ is matched with a seller $y$, they generate together the total surplus $s(x, y)$, defined as a maximum over the set of possible commodities. Then $s: X \times Y \longrightarrow[0, \infty[$ is upper semicontinuous by our assumptions (3), and the set $Z(x, y)$ where the supremum is attained (5) is non-empty, compact-valued, and upper hemicontinuous. Our normalizations (1)-(3) permit either buyer or seller to go unmatched (to match with a null type) and force the utility of the unmatched state to be zero:

$$
\begin{aligned}
& s\left(x, \emptyset_{Y}\right)=u\left(x, \emptyset_{Z}\right)=0 \\
& s\left(\emptyset_{X}, y\right)=-v\left(\emptyset_{Z}, y\right)=0 .
\end{aligned}
$$

One can then define a pairwise matching problem by the set of buyers $(X, \mu)$, the set of sellers $(Y, \nu)$, and the pairwise surplus defined by the surplus function s. An assignment (or a matching) is defined as a measure $\gamma$ on $X \times Y$, the marginals of which coincide with $\mu$ and $\nu$. Using the same notations as above, we thus write that

$$
\begin{aligned}
& \pi_{\#}^{X} \gamma=\mu \\
& \pi_{\#}^{Y} \gamma=\nu
\end{aligned}
$$

where the projection mappings $\pi^{X}(x, y)=x$ and $\pi^{Y}(x, y)=y$ this time are defined on $X \times Y$. If $(x, y) \in\left(X_{0} \times Y_{0}\right) \cap \operatorname{Spt}(\gamma)$, we say that $x$ and $y$ are matched. A buyer may be matched to multiple sellers and vice versa. If $x \in X_{0}$ and there is no $y \in Y_{0}$ such that $(x, y) \in \operatorname{Spt}(\gamma)$, we say that $x$ is unmatched (and similarly for $y$ ). 
A payoff corresponding to $\gamma$ is a pair of functions $\bar{U}: X \rightarrow \mathbf{R}$ and $\bar{V}: Y \rightarrow \mathbf{R}$ with the normalization $\bar{U}\left(\emptyset_{X}\right)=0$ such that for any $(x, y) \in \operatorname{Spt}(\gamma)$,

$$
\bar{U}(x)+\bar{V}(y) \leq s(x, y) .
$$

Finally, an outcome is defined as a triple $(\gamma, \bar{U}, \bar{V})$ where $(\bar{U}, \bar{V})$ is a payoff corresponding to $\gamma$.

We have thus showed how one can associate, to any hedonic problem, a matching model. Note that the converse is also true: for every matching problem defined by the upper semicontinuous surplus function $s(x, y)$, one can trivially construct a hedonic problem from a suitable choice of utility functions. For example $Z=Y, u=s$, with $v(y, y)=0$ and $v(x, z)=+\infty$ for all $z \neq y$. Smoother examples are more involved to articulate but also possible; in fact, every upper semicontinuous surplus function $s(x, y) \geq 0$ and continuous assignment $z(x, y)$ can be shown to arise from a hedonic model.

Stability Following the literature ${ }^{4}$, we define stability by:

Definition 2.1 An outcome $(\gamma, \bar{U}, \bar{V})$ is stable if for any $(x, y) \in X \times Y$,

$$
\bar{U}(x)+\bar{V}(y) \geq s(x, y) .
$$

Note, that this definition implies that a stable outcome satisfies

$$
\begin{aligned}
& \bar{U}(x) \geq s\left(x, \emptyset_{Y}\right)=0 \\
& \bar{V}(y) \geq s\left(\emptyset_{X}, y\right)=0
\end{aligned}
$$

for all $x \in X$ and for all $y \in Y$. In words: a match is stable if two conditions are fulfilled:

1. No matched agent would be better off unmatched.

2. No two agents $x$ and $y$, who are not matched together, would both prefer being matched together than their current situation.

To see the link between the formal and informal definitions, consider an outcome $(\gamma, \bar{U}, \bar{V})$ that satisfies (13). The functions $\bar{U}(x)$ and $\bar{V}(y)$ can be interpreted as the utilities derived by $x$ and $y$ at the outcome at stake. As noted above, restriction (13) immediately implies condition one. In addition, restriction (13) along with the definition of a payoff implies that $\bar{U}(x)+\bar{V}(y)=$ $s(x, y)$ for all $(x, y) \in \operatorname{Spt}(\gamma)$. Finally, restriction (13) guarantees that any two agents $(x, y) \notin \operatorname{Spt}(\gamma)$ who are not matched with each other, cannot generate a surplus larger than $\bar{U}(x)+\bar{V}(y)$. Indeed, if $x$ and $y$ were such that $s(x, y)>$ $\bar{U}(x)+\bar{V}(y)$, then it would be the case that (i) they are not matched together in the outcome under consideration, and (ii) they can both improve their utility by leaving their current situation and rematching together. But such a situation would violate the definition of stability.

\footnotetext{
${ }^{4}$ See for instance Roth and Sotomayor [34].
} 
Finally, a matching $\gamma$ is stable if there exists a payoff $(\bar{U}, \bar{V})$ such that the outcome $(\gamma, \bar{U}, \bar{V})$ is stable.

A well known result, in our transferable utility context, is that a matching is stable if and only if it maximizes total surplus. ${ }^{5}$ Define for each matching $\gamma$ the total surplus

$$
\gamma[s]=\int_{X \times Y} s(x, y) d \gamma(x, y) .
$$

Then

Proposition 2.2 (Gretsky, Ostroy, and Zame [18]) A matching $\gamma$ of $(X, \mu)$ with $(Y, \nu)$ is stable if and only if there exists no other matching $\gamma^{\prime}$ such that

$$
\gamma^{\prime}[s]>\gamma[s] .
$$

It follows that the matching problem is itself equivalent to a linear programming problem of the optimal transportation type, as we next discuss.

\subsection{The transportation problem}

We claim that in fact both hedonic pricing and stable matching lead to the problem of pairing buyers $(X, \mu)$ with sellers $(Y, \nu)$ so as to optimize the average (or total) of the surplus function $s(x, y)$. This problem can be expressed as a linear program:

Program (MK) (Monge-Kantorovich) Given an upper semi-continuous function $s: X \times Y \longrightarrow[0, \infty]$ on two probability spaces $(X, \mu)$ and $(Y, \nu)$, solve

$$
\max _{\gamma \in \Gamma(\mu, \nu)} \gamma[s]
$$

over the set of measures

$$
\Gamma(\mu, \nu)=\left\{0 \leq \gamma \text { on } X \times Y \mid \pi_{\#}^{X} \gamma=\mu, \quad \pi_{\#}^{Y} \gamma=\nu\right\} .
$$

with prescribed marginals. Here the $\pi$ are the projections: $\pi^{X}(x, y)=x$ and $\pi^{Y}(x, y)=y$.

Dual program $\left(\mathbf{M K}^{\prime}\right)$ This surplus maximization can be interpreted as an optimal transportation problem of Monge-Kantorovich form [30] [23] [33] [39]. The dual linear program, found by Kantorovich and his collaborators, is posed as follows. Define

$$
\mu[q]=\int_{X} q(x) d \mu(x)
$$

and

$$
\nu[r]=\int_{Y} r(y) d \nu(y) .
$$

\footnotetext{
${ }^{5}$ Shapley and Shubik [35] prove this result in the matching problem with a finite number of types. Gretsky, Ostroy, and Zame [18] extend the Shapley Shubik result to the economy with a continuum of types.
} 
Then the Kantorovich dual program is:

$$
\min _{(q, r) \in \operatorname{Lip}_{s}(\mu, \nu)}\{\mu[q]+\nu[r]\}
$$

where $\operatorname{Lip}_{s}(\mu, \nu)$ consists of all pairs of functions $q \in L^{1}(X, d \mu)$ with $q\left(\emptyset_{X}\right)=0$ and $r \in L^{1}(Y, d \nu)$ which satisfy the constraint ${ }^{6}$

$$
q(x)+r(y) \geq s(x, y) \quad \forall(x, y) \in X \times Y .
$$

Interestingly, the dual constraints (17) exactly reproduce the stability conditions (13) of the matching problem. Indeed, for any stable match, the dual variables $q(x)$ and $r(y)$ can be interpreted as a payoff.

A key property of the primal-dual pair is that for all $\gamma$ that are feasible for $(\mathrm{MK})$ and for all pairs $(q, r)$ feasible for $\left(\mathrm{MK}^{\prime}\right)$

$$
\gamma[s] \leq \mu[q]+\nu[r] .
$$

Moreover, a feasible triple $(\gamma, q, r)$ produces equality in (18) (if and) only if $\gamma$ maximizes $(\mathrm{MK})$ and the pair $(q, r)$ minimize $\left(\mathrm{MK}^{\prime}\right)$. The only if statement is obvious and plays a crucial role hereafter; the if statement is the basic duality result from linear programming (see e.g., Anderson and Nash [2], Gretsky, Ostroy and Zame [18] or Villani [39]); it can also be recovered as a special instance of the existence of a Nash equilibrium in an infinite-dimensional, two-player, zero-sum, bilinear, mixed-strategy game [42].

\section{Stable matching and hedonic pricing via opti- mal transportation}

\subsection{The matching problem: an existence result}

A first outcome of the previous arguments is a general, existence result for the optimal transportation problem, therefore for the matching problem. Specifically, the upper semi-continuity of $s(x, y) \geq 0$ guarantees the maximum (14) is attained. It has a finite value if the dual problem is feasible in which case the minimum (16) is also attained [24]. To summarize, we quote Theorem 5.9 of [41], which obviously extends from complete separable metric spaces to Borel subsets $X$ and $Y$ thereof, whose closures will be denoted $\operatorname{cl} X$ and $\operatorname{cl} Y$ :

Theorem 3.1 (Existence and duality) Let $X$ and $Y$ be Borel subsets of complete, separable metric spaces equipped with Borel probability measures $\mu$ and $\nu$. If $s: \operatorname{cl}(X \times Y) \longrightarrow[0, \infty[$ is upper semicontinuous then the maximum (14) is attained by some $\gamma \in \Gamma(\mu, \nu)$. Moreover, if some pair of real-valued

\footnotetext{
${ }^{6}$ The choice $q\left(\emptyset_{X}\right)=0$ costs no generality. Theorem 3.1 then implies $r\left(\emptyset_{Y}\right)=0$ for any pair $(q, r)$ achieving the infimum (16), in view of our normalization (2).
} 
lower semicontinuous functions $(\bar{q}, \bar{r}) \in \operatorname{Lip}_{s}(\mu, \nu)$ is feasible for the dual problem, then the minimum (16) is also attained. In this case $\gamma[s]=\mu[q]+\nu[r]<\infty$, and $\gamma$ assigns zero outer measure to the complement of the zero set

$$
S:=\{(x, y) \in X \times Y \mid q(x)+r(y)-s(x, y)=0\} .
$$

Notice our assumptions (3)-(6) on the utilities $u(x, z)$ and $v(y, z)$ imply the surplus $s(x, y)$ defined by (4) satisfies all hypotheses of this theorem.

Remark 3.2 (s-convex payoff functions) It is important to note that any feasible pair (q,r) in the minimization (16) can be replaced by $\left(r^{s}, q^{\tilde{s}}\right)$ without increase in cost, where

$$
\begin{aligned}
r^{s}(x) & =\sup _{y \in Y} s(x, y)-r(y) \\
q^{\tilde{s}}(y) & =\sup _{x \in X} s(x, y)-q(x) .
\end{aligned}
$$

Since $r^{s \tilde{s} s}=\left(\left(r^{s}\right)^{\tilde{s}}\right)^{s}=r^{s}$, it costs no generality to take $(q, r)=\left(r^{s}, q^{\tilde{s}}\right)$, meaning $q=q^{\tilde{s} s}$ and $r=r^{s \tilde{s}}$, in which case we say $q$ is $s$-convex and $r$ is $\tilde{s}$-convex [33] [39]. Then in the existence theorem above, the minimum (16) is attained by a pair of functions satisfying $(q, r)=\left(r^{s}, q^{\tilde{s}}\right)$. This fact plays a key role in the proof that the minimum is attained, in the developments to come, and in computational strategies to approximate a solution to the minimization.

The geometry of $S$, the set defined in (19) takes center stage in the analysis which follows, since this set determines which buyers can match with which sellers at equilibrium. For example, it is well known that in the one dimensional matching model with $D_{x y}^{2} s(x, y)>0$, there is a unique optimal assignment that involves positive assortative matching. In this case, the set $S$ is the graph of a strictly increasing function $y=f(x)$.

Economically, the solutions $(q, r)$ of the dual problem are also important because they represent the utility payoffs obtained by each type. Even for $x \notin \operatorname{Spt} \mu$, the range of allowed values for $q(x)$ has economic relevance, since it bounds the payoff available when a few new buyers of type $x$ choose to enter the established market; similarly, at $y \notin \operatorname{Spt} \nu$ the range of values for $r(y)$ bounds the payoff available when a few sellers of type $y$ enter the established market.

\subsection{Existence of an hedonic equilibrium}

It remains to show that the existence result obtained in the matching problem implies the existence of an hedonic equilibrium. Given the structure of the relationship between the two problems, it is clear that if buyer $x$ and seller $y$ are matched in the matching problem, they will trade some common quality $z$ in an hedonic equilibrium. What has to be constructed is a price schedule $P(z)$ that supports those trades.

Recall the definition of $Z(x, y)$ given in (5) and let $z_{0}(x, y) \in Z(x, y)$ be a measurable selection. The main result is the following: 
Proposition 3.3 (Equilibrium prices) Let $\gamma$ solve the primal program (14) and $(q, r)$ solve the dual program (16). Then there exists a Borel price function $P: Z \longrightarrow \mathbf{R} \cup\{ \pm \infty\}$ satisfying

$$
P_{\max }(z):=\inf _{y \in Y}\{v(y, z)+r(y)\} \geq P(z) \geq \sup _{x \in X}\{u(x, z)-q(x)\}=: P_{\min }(z) .
$$

With $\alpha \equiv\left(i d_{X} \times i d_{Y} \times z_{0}\right)_{\#} \gamma$, any such $P$ forms an equilibrium pair $(\alpha, P)$.

Proof. Combining (17) with the definition of the surplus $s$ :

$$
q(x)+r(y) \geq s(x, y) \geq u(x, z)-v(y, z) \quad \text { on } X \times Y \times Z,
$$

hence

$$
v(y, z)+r(y) \geq u(x, z)-q(x) \quad \text { on } X \times Y \times Z
$$

and

$$
\inf _{y}\{v(y, z)+r(y)\} \geq \sup _{x}\{u(x, z)-q(x)\} \quad \text { on } Z .
$$

Choose any Borel function $P(z)$ satisfying (22); the infimum or supremum themselves would suffice.The basic duality result from linear programming asserts $\gamma[s]=\mu[q]+\nu[r]=\gamma[q+r]$, since $\gamma \geq 0$ has $\mu$ and $\nu$ for marginals. Thus equality holds for $\gamma$-a.e. $(x, y)$ in the inequality (17); i.e. whenever $\gamma$ matches buyer $x$ with seller $y$. Consider $\bar{x}$ who is matched with $\bar{y}$, in the sense that they belong to the Borel set $S$ defined in (19). This is the set of full $\gamma$ measure where equality holds in the dual inequality constraints. Since the pair $(\bar{x}, \bar{y})$ agree on their preferred contracts $\bar{z} \in Z(\bar{x}, \bar{y})$ attaining (4),

$$
q(\bar{x})+r(\bar{y})=s(\bar{x}, \bar{y})=u(\bar{x}, \bar{z})-v(\bar{y}, \bar{z})
$$

and we have

$$
v(\bar{y}, \bar{z})+r(\bar{y})=P(\bar{z})=u(\bar{x}, \bar{z})-q(\bar{x})
$$

on the set $T:=\{(\bar{x}, \bar{y}, \bar{z}) \in X \times Y \times Z \mid \bar{z} \in Z(\bar{x}, \bar{y}))\}$. Upper hemicontinuity of $Z(\bar{x}, \bar{y})$ implies $T$ is closed, while $z_{0}(\bar{x}, \bar{y}) \in Z(\bar{x}, \bar{y})$ implies Spt $\alpha \subset T$. Our choice (22) of price now yields

$$
u(\bar{x}, z)-P(z) \leq q(\bar{x})=u(\bar{x}, \bar{z})-P(\bar{z}) \quad \forall z \in Z
$$

and

$$
P(z)-v(y, z) \leq r(\bar{y})=P(\bar{z})-v(\bar{y}, \bar{z}) \quad \forall z \in Z
$$

so that $\bar{z}$ maximizes both $u(\bar{x}, z)-P(z)$ and $P(z)-v(\bar{y}, z)$. Since the equalities hold for $(\bar{x}, \bar{y}, \bar{z})$ in a set $(S \times Z) \cap \operatorname{Spt} \alpha$ of full measure for $\alpha$, we conclude that $(\alpha, P)$ is a market-clearing hedonic equilibrium pair.

The left side of $(22), P_{\max }(z)$, is the minimum equilibrium willingness to accept of all sellers in the market. No sellers will trade $z$ unless $P(z) \geq P_{\max }(z)$. The right side of $(22), P_{\min }(z)$, is the maximum equilibrium willingness to pay of all buyers. No buyers will trade $z$ unless $P(z) \leq P_{\min }(z)$. When $P_{\max }(z)>$ 
$P_{\min }(z)$ no trade takes place. When $P_{\max }(z)=P_{\min }(z)$, an exchange may be made by the set of buyers and sellers who attain the infimum and supremum.

The result implies that to any stable match corresponds an hedonic equilibrium. Therefore, the existence result derived in the previous section has the immediate, following consequence:

Corollary 3.4 The hedonic model described in Section 1 has an equilibrium.

It is important to note that existence obtains in a general context. No restriction is imposed on the dimension of the spaces at stake nor on the measures describing the distributions of types. Both discrete and continuous distributions are allowed. Moreover, no specific assumptions are made on $u$ and $v$ beyond the standard ones. In particular, we do not assume any Spence-Mirrlees condition. Our result thus establishes the existence of hedonic equilibria in a fully general context. $^{7}$

Finally, it is interesting to note that the converse is also true: to any hedonic equilibrium, one can associate a stable match, as asserted by the following result:

Lemma 3.5 Let $(\alpha, P)$ be a hedonic equilibrium pair. Then the indirect utilities $U(x)$ and $V(y)$ from (7)-(8) minimize Kantorovich's dual problem (16), while $\gamma=\left(\pi^{X} \times \pi^{Y}\right)_{\#} \alpha$ maximizes the primal problem (14). Here $\pi^{X}(x, y, z)=x$ and $\pi^{Y}(x, y, z)=y$.

Proof. First observe that equilibrium condition (9) states that $\gamma$ has $\mu$ and $\nu$ for marginals, hence is a feasible competitor in the Monge-Kantorovich primal program (14). The definitions (7) and (8) of $U(x)$ and $V(y)$ imply

$$
U(x)+V(y) \geq u(x, z)-v(y, z)
$$

for all $z \in Z$. Taking the supremum over $z \in Z$ implies that $(U, V)$ is a feasible pair for the Kantorovich dual program (16). Moreover, equilibrium condition (10) forces equality in (23) for $\alpha$-a.e. $(x, y, z) \in \operatorname{Spt}(\alpha)$, hence

$$
U(x)+V(y)=s(x, y) .
$$

The lower bounds $U, V \geq 0$ permit this to be integrated against $\alpha$, yielding

$$
\int U(x) d \mu(x)+\int V(y) d \nu(y)=\int s(x, y) d \gamma(x, y) .
$$

Hence $\gamma$ maximizes the primal program whilst the pair $(U, V)$ minimizes the dual program.

In other words, the hedonic pricing problem with quasilinear utility, the stable matching problem with transferable utility and the optimal transportation

\footnotetext{
${ }^{7}$ Ekeland [12], [13] presents an alternative proof based on convex programming instead of linear programming. Gretsky, Ostroy, and Zame [18] prove existence in a version of the model in which sellers are endowed with $z$. That is, $v(y, z)=+\infty$ unless $y=z$, so seller utility is simply $v(z)$.
} 
problem are equivalent; none is more nor less general than the others. Moreover, approximate solutions can be computed using linear programming techniques. This opens up the study of these problems in empirical settings in which the type spaces are high dimensional, have both discrete and continuous elements, and have different dimensions on the buyer and seller side of the market. More work needs to be done to study these problems in these applied settings.

In theoretical settings, one obtains necessary and sufficient conditions for the optimal assignment or the stable matching, now shown to exist, via the KuhnTucker conditions from linear programming. The form of these conditions in the optimal transportation context is well-understood [33] [41]. In a suitably weak topology, one could also show that the solution depends continuously on the data in the sense that the limit of a sequence of solutions to different problems is a solution of the limiting problem. However, to make concrete statements about uniqueness of the solution or the form of the optimal measure $\gamma$, requires additional structure on the problem. This is the topic of the next section.

\section{Uniqueness and purity}

\subsection{Pure solutions}

We consider two properties of the equilibrium, namely uniqueness and purity. Gretsky, Ostroy and Zame [18] study whether the dual has unique solutions; i.e. whether the equilibrium payoffs to agents are unique, and derive a condition equivalent to this in terms of differentiability of the social gains function, the value of the primal program. This gains function is endogenous. In the smooth setting, we complement their result by giving sufficient conditions for uniqueness of marginal payoffs in terms of exogenous parameters of the hedonic model. We then focus our attention on whether the measure describing the matching of buyers to sellers is unique. Purity of the equilibrium is concerned with whether the equilibrium strategies of buyers and sellers are pure or mixed. Intuitively, an equilibrium is pure if (almost) all agents have a pure strategy at equilibrium - i.e., for each agent there exists one trading partner that she chooses with probability one. In the opposite case of a non-pure equilibrium, a non-null set of agents are either indifferent between several partners, or indifferent between action and inaction; then equilibrium may require randomization or mixed strategies.

A couple of well-known examples illustrate these ideas. We start with a standard situation in which the Spence-Mirrlees condition guarantees a unique, pure equilibrium.

Example 4.1 (Positive assortative matching) Consider a hedonic economy in which $X_{0}=Y_{0}=Z_{0} \subseteq \mathbf{R}$. If $s(x, y)$ is differentiable and $D_{x y}^{2} s(x, y)>0$, then there is a unique equilibrium, it involves positive assortative matching, and all but countably many agents have a pure strategy optimum. The same facts are true if $s(x, y)$ is supermodular. Both $D_{x y}^{2} s(x, y)>0$ and supermodularity of $s(x, y)$ are versions of a Spence-Mirrlees or single-crossing condition. 
At the opposite end of the spectrum, we may have models in which a continuum of (pure and non-pure) equilibria exist:

Example 4.2 (Orthogonal surplus) Consider a plane, and let $X_{0}$ be the interval $[0,1]$ on the horizontal axis, and $Y_{0}$ be the interval $\left[-\frac{1}{2}, \frac{1}{2}\right]$ on the vertical axis; both sets are equipped with the uniform distribution. Finally, consider the surplus $s(x, y)=2-x^{2}-y^{2}$; i.e., any match generates a surplus of two, from which a transportation cost equal to the distance between the two points is withdrawn. Then the maximum aggregate surplus, equal to $5 / 12$, is obtained by uncountably many measures $\gamma$, including pure solutions (e.g., the uniform distribution over the graph of functions like $f(x)=1 / 2-x$ or $f(x)=x-1 / 2)$ and nonpure solutions (e.g. the uniform distribution over the square $\left.[0,1] \times\left[-\frac{1}{2}, \frac{1}{2}\right]\right)$.

These examples suggest interesting conclusions. First, additional restrictions are clearly needed to guarantee either uniqueness or purity. In the first example, a standard Spence-Mirrlees condition produces assortative matching, which in turn guarantees uniqueness and purity. Note, however, that in this example (as in the second one) the sets $X_{0}$ and $Y_{0}$ are one-dimensional.

When we move away from the one dimensional matching model, the concept of assortative matching is not well-defined. In such economies, in which $X_{0}$ and $Y_{0}$ are not subsets of the real line and the surplus $s$ need not be differentiable, a condition more general than the Spence-Mirrlees conditions above is required. In this section we recall such a condition. The generalized Spence-Mirrlees condition (or 'twist condition', as it is known in the mathematics literature) is sufficient for both uniqueness and purity. We develop a version of this condition that is valid in general type spaces, does not require differentiability of the surplus function, allows for non-participation, and is not dependent on the coordinates (i.e. the parametrization) of the problem. This condition also need only apply to either the buyers or the sellers.

Finally, we emphasize that uniqueness and purity are different concepts. For instance, a unique equilibrium may fail to be pure, as we illustrate in an example. Therefore, we introduce a condition (called subtwist below) weaker than generalized Spence-Mirrlees, and we show that this condition is sufficient for uniqueness but not purity. Let us define the concept of pure matchings formally.

Definition 4.3 (Pure) Let $X=X_{0} \cup\left\{\emptyset_{X}\right\}$ and $Y=Y_{0} \cup\left\{\emptyset_{Y}\right\}$ be Borel subsets of complete, separable metric spaces augmented with isolated points $\emptyset_{X}, \emptyset_{Y}$, and equipped with Borel probability measures $\mu$ and $\nu$. A feasible (but not necessarily optimal) solution $\gamma \in \Gamma(\mu, \nu)$ to (MK) program (14) is pure if there exists a function $f: X_{0} \longrightarrow Y$ such that $\gamma$ is concentrated on the graph of $f$, in the sense that $\gamma$ assigns zero outer measure to the set $\left\{(x, y) \in X_{0} \times Y \mid y \neq f(x)\right\}$.

In words, if the solution is pure, then there exists a well-defined function $f$ such that any $x \in X_{0}$ is matched with probability one to $y=f(x)$. The set of buyers who remain indifferent between action or inaction, or between two or more preferred sellers, forms a set of measure zero; almost every buyer has 
a pure (as opposed to mixed) preference for whether he wishes to buy, and if so from whom. Such a pure solution will entail a pure matching of buyers and sellers to products if $Z(x, f(x)):=\underset{z \in Z}{\arg \max }\{u(x, z)-v(f(x), z)\}$ is a singleton. Note that most empirical studies consider only solutions which are pure, and for which $Z(x, f(x))$ consists of a single contract (for $\mu$ almost all $x \in X_{0}$ ).

\subsection{A generalized Spence-Mirrlees (twist) condition}

A standard tool in economic approaches of matching or hedonic problems is the Spence-Mirrlees condition - also known as the twist condition in the mathematics literature [41]. Though the Spence-Mirrlees conditions has been generalized to multidimensional type spaces - see Gangbo [14], Carlier [8], or Ma, Trudinger, and Wang [27] - one may notice that the vast majority of economic studies still adopt a one-dimensional version of the condition.

Let us first specialize to the Lipschitz-buyer setting, meaning the space of buyers $X_{0}$ is an $n$-dimensional manifold (smooth without loss of generality), and the surplus function $s(x, y)$ and distribution $d \mu_{0}(x)$ of buyers enjoy a sufficiently smooth dependence on $x \in X_{0}$, as we now make precise. We describe this setting as Lipschitz-buyer to emphasize that $Y_{0}$ and $Z_{0}$ may or may not be smooth manifolds, and could even be finite spaces as when a continuum of buyers match with finitely many sellers. In this and subsequent definitions (of the twist and subtwist conditions, and of numbered limb systems), we go to some trouble to define notions which are independent of local choices of coordinates on the manifold $X_{0}$. The reason for this is the following. Imagine a model which matches workers with varying skill levels $x \in X_{0}$ with firms which employ different technologies $y \in Y_{0}$. Obviously the skill level of the workers can be assessed (or parameterized) in many different ways. However, the question of whether the surplus function $s(x, y)$ is Lipschitz, semiconvex, twisted or subtwisted should be independent of the methodology used to assess the worker's skill levels, at least among methodologies which provide equivalent information. This principle of parametrization independence also plays a striking role in the regularity theory [25]. The reader may prefer to skip the formal definitions, consulting instead the examples of relevance immediately thereafter.

Definition 4.4 (Lipschitz and semiconvex functions) Let $X_{0}$ be a smooth $n$-dimensional manifold and $X=X_{0} \cup\left\{\emptyset_{X}\right\}$. Then $s: X \times Y \longrightarrow \mathbf{R}$ is said to be Lipschitz on $X_{0}$ uniformly in $Y$ if for each (nonmaximal) coordinate ball $B_{R} \subset X_{0} \backslash \partial X_{0}$, there is a constant $C_{B}$ depending on the coordinates and the ball, but independent of $y$, such that all $x, \bar{x} \in B_{R}$ satisfy

$$
|s(x, y)-s(\bar{x}, y)| \leq C_{B}\|x-\bar{x}\|,
$$

where $\|x-\bar{x}\|$ denotes the distance in coordinates. Similarly, $s(x, y)$ is semiconvex on $X_{0}$ uniformly in $Y$ if for each (nonmaximal) coordinate ball $B_{R} \subset$ $X_{0} \backslash \partial X_{0}$, there is a constant $C_{B}$ depending on the coordinates and the ball, but independent of $y \in Y$, such that

$$
x \in B_{R} \longrightarrow s(x, y)+C_{B}\|x\|^{2} / 2
$$


is a convex function in these coordinates. A function $q: X \longrightarrow \mathbf{R}$ is said to be Lipschitz on $X_{0}$, or semiconvex on $X_{0}$, if $s(x, y)=q(x)$ independent of $y \in Y$ satisfies the corresponding definitions above.

It might be appropriate to add the adjective local to the definitions of Lipschitz and semiconvex given above, but since differentiable manifolds are defined by local charts, there is no good definition for what it might mean for a function thereon to be globally Lipschitz, so we omit the adjective local for brevity whenever we feel confusion cannot arise. For this purpose, we overlook the fact that $X_{0}$ was assumed to be a metric space at the outset.

Definition 4.5 (Lipschitz-buyer and semiconvex-buyer settings) Let $X_{0}$ be a smooth $n$-dimensional manifold and $\mu$ a Borel probability measure on $X:=$ $X_{0} \cup\left\{\emptyset_{X}\right\}$. The setting is Lipschitz-buyer if $\mu$ concentrates no mass on subsets of $X_{0}$ which have zero volume, and if moreover the surplus function $s \in C(X \times Y)$ is locally Lipschitz on $X_{0}$ uniformly in $Y$. Similarly, the setting is semiconvexbuyer if $\mu$ concentrates no mass on ( $C^{2}$-rectifiable) hypersurfaces in $X_{0}$, and if the surplus function $s \in C(X \times Y)$ is locally semiconvex on $X_{0}$ uniformly in $Y$.

As the next examples show, our model falls into the semiconvex-buyer setting whenever the buyer's utility $u(x, z)$ or the surplus function $s(x, z)$ is sufficiently smooth. Although its description appears more technical, the semi-convex buyer setting has the advantage that the measure $\mu$ may be more concentrated than the Lipschitz-buyer setting allows. In particular, a measure $\mu_{0}$ on the interval $X_{0}=$ $[0,1]$ satisfies the semiconvex-buyer hypothesis as long as it assigns zero mass $\mu_{0}(\{x\})=0$ to each type $x \in[0,1]$; it need not be absolutely continuous with respect to Lebesgue measure, as the Lipschitz-buyer hypothesis would require. This improvement goes back to Gangbo and McCann [28] [16], though we also exploit Veselý and Zajíček's implicit function theorem for convex differences [38] to deduce that a semiconvex function $q: X_{0} \longrightarrow \mathbf{R}$ must be differentiable everywhere outside of a $C^{2}$-rectifiable hypersurface in $X_{0}$.

Example 4.6 (Lipschitz-buyer) If $X_{0}$ is a smooth manifold and $Y$ is compact, any surplus function $s(x, y)$ locally Lipschitz on $X_{0} \times Y$ also satisfies (24). Similarly if $X_{0}$ is a smooth manifold, $Z$ is compact, and $v: Y \times Z \longrightarrow \mathbf{R}$ is arbitrary, any utility function $u(x, z)$ locally Lipschitz on $X_{0} \times Z$ induces a surplus (4) satisfying the Lipschitz-buyer hypothesis (24).

Example 4.7 (Semiconvex-buyer) If $X_{0}$ is a smooth manifold and $Y$ is compact, any surplus function $s \in C^{2}\left(X_{0} \times Y\right)$ also satisfies (25). If $X_{0}$ is a smooth manifold, $Z$ is compact, and $v: Y \times Z \longrightarrow \mathbf{R}$ is arbitrary, any utility function $u \in C^{2}\left(X_{0} \times Z\right)$ induces a surplus (4) satisfying the semiconvex-buyer hypotheses, despite the fact that $s(x, y)$ will not generally be differentiable.

Although the surplus function (4) may fail to be differentiable, the Lipschitzbuyer setting guarantees the surplus $s(x, y)$ is locally Lipschitz ${ }^{8}$ with respect to

\footnotetext{
${ }^{8}$ See Theorem 10.26 of [41]. The same technique validates the claims made in Examples 4.6-4.7.
} 
$x \in X_{0}$, with Lipschitz constant independent of $y \in Y$. This in turn guarantees any $s$-convex function $q=q^{\tilde{s} s}$ will be locally Lipschitz on $X_{0}$, hence (by Rademacher's theorem) differentiable on a set Dom $D q \subset X_{0}$ of full measure. The derivative $D q(x)$ is a vector in the cotangent space $T_{x}^{*} X_{0}$ to $X_{0}$ at the point $x \in \operatorname{Dom} D q$. Given $q: X_{0} \longrightarrow \mathbf{R}$ locally Lipschitz, we define its superdifferential $\partial q\left(x_{0}\right)$ at $x_{0} \in X_{0}$ to consist of the set of covectors $w \in T_{x_{0}}^{*} X_{0}$ such that

$$
q(x) \leq q\left(x_{0}\right)+\left\langle w, x-x_{0}\right\rangle+o\left(\left|x-x_{0}\right|\right) \quad \text { as } \quad x \rightarrow x_{0} .
$$

For fixed $y \in Y$, we define the superdifferential $\partial^{x} s\left(x_{0}, y\right) \subset T_{x_{0}}^{*} X$ of $s(x, y)$ with respect to $x$ analogously.

Before proceeding, let us state a uniqueness proposition which does not require further assumptions. This proposition asserts $\mu$-a.e. uniqueness of the marginal payoff $D q$ with respect to buyer type, which may or may not determine the payoff $q(x)$ uniquely depending on the connectivity properties of Spt $\mu_{0} \subset$ $X_{0}$, and whether the participation constraint is active. Still, this proposition yields a point of contact between our work and that of Gretsky, Ostroy and Zame [19], by giving sufficient conditions on the measures and surplus function to enforce their uniqueness hypothesis. Note however, in the absence of further assumptions such as the twist condition from Definition 4.9, our proof of this proposition may not extend to the Lipschitz-buyer setting. Our proposition is inspired by a pressure uniqueness result of Brenier concerning fluid mechanics [6].

Proposition 4.8 (A semiconvex buyer's marginal payoffs are unique) Let $s: X \times Y \longrightarrow[0, \infty[$ be defined on probability spaces $(X, \mu)$ and $(Y, \nu)$ in the semiconvex-buyer setting. If both $(q, r)$ and $(\tilde{q}, \tilde{r}) \in \operatorname{Lip}_{s}(\mu, \nu)$ minimize (16), and both $q$ and $\tilde{q}$ are (locally) semiconvex, then $D q=D \tilde{q}$ holds $\mu$-almost everywhere on $X \backslash\left\{\emptyset_{X}\right\}$.

Proof. Suppose $(q, r) \in \operatorname{Lip}_{s}(\mu, \nu)$ minimizes (16). Then (21) implies $q^{\tilde{s}} \leq r$ hence $\left(q, q^{\tilde{s}}\right) \in \operatorname{Lip}_{s}(\mu, \nu)$ also minimizes (16), and it costs no generality to assume $r=q^{\tilde{s}}$ as in Remark 3.2. Moreover, $r$ is lower semicontinuous in (21) by the continuity assumption $s \in C(X \times Y)$. Let

$$
S:=\{(x, y) \in X \times Y \mid q(x)+r(y)-s(x, y)=0\} .
$$

denote the closed set where the lower semicontinuous non-negative function $q(x)+r(y)-s(x, y)$ vanishes. Since $\mu$ concentrates no mass on the $\left(C^{2}\right.$ rectifiable $)$ hypersurfaces where differentiability of $q$ fails, all joint measures $\gamma \in \Gamma(\mu, \nu)$ assign full mass to $R=\operatorname{Dom} D q \times Y$ in the semiconvex-buyer setting, noting the convention $\emptyset_{X} \in \operatorname{Dom} D q$. Moreover, at least one optimizer $\gamma \in \Gamma(\mu, \nu)$ exists, and its support is contained in the closed set $S$, according to Theorem 3.1.

If $X$ and $Y$ are complete separable metric spaces, let $K$ denote the $\sigma$-compact carrying the full mass of $\gamma$ provided e.g., by p. 255 of [11] or Theorem I-55 of [40], so that $\mu$ vanishes outside the $\sigma$-compact projection of $K \cap \operatorname{Spt} \gamma$ through $\pi^{X}$. Now suppose $x_{0} \in \pi^{X}(K \cap \operatorname{Spt} \gamma) \cap \operatorname{Dom} D q \backslash\left\{\emptyset_{X}\right\}$. Then there exists 
$\left(x_{0}, y_{0}\right) \in \operatorname{Spt} \gamma \subset S$, whence the first-order condition for vanishing in (27) implies superdifferentiability of $s\left(x, y_{0}\right)$ at $x_{0}$ with $D q\left(x_{0}\right) \in \partial^{x} s\left(x_{0}, y_{0}\right)$. On the other hand, semiconvexity implies subdifferentiability and hence differentiability of $x \in X_{0} \longrightarrow s\left(x, y_{0}\right)$ at $x_{0}$, and its super- and subdifferentials must both then coincide with $\left\{D_{x} s\left(x_{0}, y_{0}\right)\right\}$, as in [16]. Thus $D q\left(x_{0}\right)=D_{x} s\left(x_{0}, y_{0}\right)$. Notice the right hand side depends only on $\left(x_{0}, y_{0}\right) \in \operatorname{Spt} \gamma$ such that $x_{0} \in \pi^{X}(K \cap \operatorname{Spt} \gamma) \cap$ Dom $D q \backslash\left\{\emptyset_{X}\right\}$, and is otherwise independent of $q$. If a second semiconvex function $\tilde{q}$ minimized (16), we would similarly have $D \tilde{q}\left(x_{0}\right)=D_{x} s\left(x_{0}, y_{0}\right)=$ $D q\left(x_{0}\right)$ on the set $\pi^{X}(K \cap \operatorname{Spt} \gamma) \cap \operatorname{Dom} D q \cap \operatorname{Dom} D \tilde{q}$ of full $\mu_{0}$ measure, to establish the proposition.

If $X$ and $Y$ are merely Borel subsets of complete separable metric spaces, we use their completions $\tilde{X}$ and $\tilde{Y}$ to find a $\sigma$-compact set $\tilde{K} \subset \tilde{X} \cap \tilde{Y}$ carrying the full mass of $\gamma$, and establish the result on the intersection of the $\sigma$-compact set $\pi^{\tilde{X}}(\tilde{K} \cap \operatorname{Spt} S)$ with Dom $D q \subset X$, which still carries the full mass of $\mu$.

We now state a generalization of the Spence-Mirrlees condition appropriate to the Lipschitz-buyer setting.

Definition 4.9 (Twisted-buyer condition) In the Lipschitz-buyer setting, a surplus function $s: X \times Y \longrightarrow[0, \infty[$ is said to be twisted-buyer if there is a set $X_{L} \subset X_{0}$ of zero volume such that $\partial^{x} s\left(x_{0}, y_{1}\right)$ is disjoint from $\partial^{x} s\left(x_{0}, y_{2}\right)$ for all $x_{0} \in X_{0} \backslash X_{L}$ and $y_{1} \neq y_{2}$ in $Y$.

Example 4.10 A surplus differentiable with respect to $x$ is twisted-buyer if and only if there is a negligible set $X_{L} \subset X_{0}$ of buyers such that: for each distinct pair of sellers, any critical points of the function $x \in X_{0} \longrightarrow s\left(x, y_{1}\right)-s\left(x, y_{2}\right)$ lie in $X_{L}$.

For instance, the surplus function $s(x, y)=2-|x-y|^{2}$ on disjoint open sets $X_{0}, Y_{0} \subset \mathbf{R}^{n}$ is both twisted-buyer and twisted-seller. We must insist on disjointness of $X_{0}$ and $Y_{0}$ since for $y \in X_{0}$ the function $x \in X_{0} \longrightarrow s(x, y)-$ $s\left(x, \emptyset_{Y}\right)=2-|x-y|^{2}$ has $x=y$ as a critical point. This fact has been exploited in matching problems with optional participation, as in Caffarelli \& McCann [7]. On the other hand, with the surplus function of Example 4.2, neither the twisted-buyer nor the twisted-seller condition is satisfied, since $s\left(x, y_{1}\right)-$ $s\left(x, y_{2}\right)=\left(y_{2}\right)^{2}-\left(y_{1}\right)^{2}$ does not depend on $x$.

The twisted-buyer condition has two consequences which are well-known (see [8], [14], or [27]) provided that at equilibrium, participation is complete. It guarantees the Monge-Kantorovich maximization (14) is attained by a unique assignment $\gamma$ of buyers with sellers. Moreover, it also implies this unique maximizer is pure, meaning there is a mapping $f: X \rightarrow Y$ defined $\mu$-almost everywhere such that $\gamma=\left(i d_{X} \times f\right)_{\#} \mu$. The following theorem confirms that the twisted-buyer condition formulated above guarantees uniqueness and purity of the mixed solution even when the situation is complicated by the presence of the isolated point $\emptyset_{X}$ in $X=X_{0} \cup\left\{\emptyset_{X}\right\}$ representing the null buyer. The proof makes use of Theorem A.3 from the Appendix which allows us to establish a unique representation of the equilibrium measure simply by showing that almost 
all sellers have pure preferences at equilibrium. We recall the full proof not only for the sake of completeness but to illustrate the efficacy of Theorem A.3.

Theorem 4.11 (Twisted-buyers induce pure and unique assignments) Let $s: X \times Y \longrightarrow[0, \infty[$ be a twisted-buyer surplus function, defined on probability spaces $(X, \mu)$ and $(Y, \nu)$ in either the Lipschitz-buyer or semiconvex-buyer setting. Then the maximizer $\gamma$ of (14) on $\Gamma(\mu, \nu)$ is unique. Moreover, there is a $\mu_{0}$ measurable map $f: X_{0} \longrightarrow Y$ such that $\gamma=\gamma_{0}+\gamma_{1}$ where $\gamma_{1}=\left(i d_{X_{0}} \times f\right)_{\#} \mu_{0}$ and $\gamma_{0}=\left(\emptyset_{X} \times i d_{Y}\right)_{\#}\left(\nu-\pi_{\#}^{Y} \gamma_{1}\right)$.

Proof. Theorem 3.1 and Remark 3.2 provide a non-negative $s$-convex minimizing pair $(q, r)=\left(r^{s}, q^{\tilde{s}}\right)$ to the dual problem (16). Recall that $q$ is then locally Lipschitz on $X_{0}$, by e.g. Theorem 10.26 [41], and $r=q^{\tilde{s}}$ is Borel in (21) by the continuity assumption $s \in C(X \times Y)$. The same theorem shows $q$ to be locally semiconvex in the semiconvex-buyer setting. Let

$$
S:=\{(x, y) \in X \times Y \mid q(x)+r(y)-s(x, y)=0\} .
$$

denote the Borel set where the non-negative function $q(x)+r(y)-s(x, y)$ vanishes. Since $\mu$ concentrates no mass on subsets of zero volume and $X_{0} \backslash$ Dom $D q$ has zero volume, all joint measures $\gamma \in \Gamma(\mu, \nu)$ assign full mass to $R=\left(\operatorname{Dom} D q \backslash X_{L}\right) \times Y$ in the Lipschitz-buyer setting, with the convention $\emptyset_{X} \in \operatorname{Dom} D q$. The same conclusion is true in the semiconvex-buyer setting, since $\mu$ is then assumed to vanish on the $\left(C^{2}\right.$-rectifiable) hypersurface where differentiability of $q$ fails. Moreover, all optimizers $\gamma \in \Gamma(\mu, \nu)$ vanish outside $S$, according to Theorem 3.1. The remainder of the proof will be devoted to showing that $S \cap R$ is contained in a numbered limb system as defined in Definition A.2. Once this is established, Theorem A.3 asserts there is only one measure in $\Gamma(\mu, \nu)$ that vanishes outside $S \cap R$, hence the optimizer is unique.

Set $I_{3}=X_{0}, I_{2}=Y$ and $I_{1}=\left\{\emptyset_{X}\right\}$ and $I_{0}=\emptyset=\operatorname{Dom} f_{1}$. Set $f_{2}(y)=\emptyset_{X}$ for all $y \in \operatorname{Dom} f_{2}=Y$. It remains only to show that $\pi^{X}(x, y)=x$ gives an injective map from $S_{3}:=(S \cap R) \cap\left(X_{0} \times Y\right)$ to Dom $f_{3}:=\pi^{X}\left(S_{3}\right)$. Once this injectivity has been shown, $f_{3}$ can be defined to make $i d_{X} \times f_{3}$ : Dom $f_{3} \longrightarrow$ $S_{3}$ invert $\left.\pi^{X}\right|_{S_{3}}$, and a comparison with Definition A.2 then reveals that $S$ is contained in a numbered limb system. The $\mu_{0}$-measurability of $f_{3}: \operatorname{Dom} f_{3} \longrightarrow$ $Y$ and special form $\gamma=\gamma_{3}+\gamma_{2}$ with $\gamma_{3}=\left(i d_{X_{0}} \times f\right)_{\#} \mu_{0}$ and $\gamma_{2}=\left(\emptyset_{X} \times\right.$ $\left.i d_{Y}\right)_{\#}\left(\nu-\pi_{\#}^{Y} \gamma_{3}\right)$ both follow from Theorem A.3.

To prove the required injectivity, suppose $\left(x_{0}, y_{1}\right)$ and $\left(x_{0}, y_{2}\right)$ both belong to $S_{3} \subset S \cap R$. The function $q(x)+r(y)-s(x, y) \geq 0$ vanishes at all points in $S$, hence enjoys $\mathbf{0}$ as a subgradient there. If this function is differentiable with respect to $x$ at $x_{0} \in \operatorname{Dom} D q \backslash X_{L}$, we have $D_{x} s\left(x_{0}, y_{1}\right)=D q(x)=D_{x} s\left(x_{0}, y_{2}\right)$; otherwise $D q\left(x_{0}\right) \in \partial^{x} s\left(x_{0}, y_{1}\right) \cap \partial^{x} s\left(x_{0}, y_{2}\right)$. In either case, the twisted-buyer condition yields $y_{1}=y_{2}$, whence $\pi^{X}$ is injective on $S_{3}$ and the proof is complete.

As anticipated, the set (19) takes center stage in the foregoing analysis. 


\subsection{Examples of twisted-buyer costs}

Since the surplus depends on the utility functions, it is useful to have criteria on $u(x, z)$ and $v(y, z)$ which guarantee $s(x, y)$ is twisted. One such criterion is given by the following example.

Example 4.12 Consider the Tinbergen (1956) model. Here, $X=Y=Z=\mathbf{R}^{n}$ with $u(x, z)=\frac{1}{2}(x-z)^{\prime} A(x-z)$ and $v(y, z)=\frac{1}{2}(y-z)^{\prime} B(y-z)$ with $A$ and $B$ symmetric and with $A-B<0$. Then

$$
s(x, y)=\frac{1}{2}\left(x^{\prime} A x-y^{\prime} B y\right)-\frac{1}{2}(A x-B y)^{\prime}(A-B)^{-1}(A x-B y)
$$

and

$$
D_{x}\left(s\left(x, y_{1}\right)-s\left(x, y_{2}\right)\right)=-\frac{1}{2}(A-B)^{-1} B\left(\left(y_{1}-y_{2}\right)\right) A^{\prime} .
$$

This only equals zero when $y_{1}=y_{2}$ so $s(x, y)$ satisfies the both buyer and seller twist condition. is

In this model, the hedonic equilibrium is unique. Buyers' willingness to pay

$$
W T P=\frac{1}{2}(x-z)^{\prime} A(x-z)-q(x) .
$$

If $A<0$ and $B>0$, buyers with smaller values of $|x-z|$ are willing to pay more for $z$ and sellers with smaller values of $|y-z|$ are willing to accept more. The willingness to pay curves of different buyers never cross. The exact balance of buyers and sellers across locations depends on the distributions of buyer and seller types in the economy.

As a more general example in which the twist condition is satisfied, consider the following lemma.

Lemma 4.13 (Utilities yielding a twisted surplus) Let $X_{0}, Y_{0}, Z_{0} \subseteq \mathbf{R}^{n}$ be open domains with $X_{0}$ and $Y_{0}$ convex. Take $u \in C^{2}\left(X_{0} \times Z_{0}\right)$ and $v \in$ $C^{2}\left(Y_{0} \times Z_{0}\right)$ with $D_{x} u(x, z) \neq \mathbf{0}$ on $X_{0} \times Z_{0}$. For $\mu_{0} \times \nu_{0}$ a.e. $(x, y)$, assume $Z(x, y)=\arg \max _{z \in Z}\{u(x, z)-v(y, z)\}=\left\{z_{0}(x, y)\right\}$ is a singleton, and for all $(x, y) \in X_{0} \times Y_{0}$ assume $M+M^{t}>0$, where

$$
M=D_{x z}^{2} u\left(x, z_{0}\right)\left(D_{z z}^{2} u\left(x, z_{0}\right)-D_{z z}^{2} v\left(y, z_{0}\right)\right)^{-1} D_{z y}^{2} v\left(y, z_{0}\right)
$$

and $z_{0}=z_{0}(x, y)$. Then $s(x, y)$ satisfies the twisted-buyer condition.

Proof. Ignoring $\emptyset_{Z}$, the surplus function is given by

$$
s(x, y)=\max _{z \in Z_{0}}\{u(x, z)-v(y, z)\} .
$$

Since $z_{0}(x, y)$ is unique and on the interior of $Z_{0}$ by assumption, and since $u$ and $v$ are differentiable, $z_{0}(x, y)$ satisfies

$$
D_{z} u\left(x, z_{0}\right)-D_{z} v\left(y, z_{0}\right)=0 .
$$


The envelope theorem then implies differentiability of $s$ at $(x, y)$, and

$$
D_{x} s(x, y)=D_{x} u\left(x, z_{0}(x, y)\right) .
$$

If for each $x \in X_{0}, y \in Y \longrightarrow D_{x} s(x, y)$ is injective, then $s(x, y)$ satisfies the twist condition. Since $Y$ is convex, a sufficient condition for this is the positive (or negative) definiteness of the quadratic form $D_{x y}^{2} s(x, y)$. Since $z_{0}=$ $z_{0}(x, y)$ maximize surplus, $D_{z z}^{2} u\left(x, z_{0}\right)-D_{z z}^{2} v(y, z) \leq 0$, and the inequality is strict by hypothesis. The implicit function theorem then implies continuous differentiability of $z_{0}(x, y)$ in (28). Differentiating (29) with respect to $y$ yields

$$
D_{x y}^{2} s(x, y)=D_{x z}^{2} u\left(x, z_{0}(x, y)\right) D_{y} z_{0}(x, y),
$$

while differentiating (28) with respect to $y$ yields

$$
\left(D_{z z}^{2} u\left(x, z_{0}\right)-D_{z z}^{2} v\left(y, z_{0}\right)\right) D_{y} z_{0}(x, y)-D_{z y}^{2} v\left(y, z_{0}\right)=0 .
$$

These combine to give

$$
D_{y} z_{0}(x, y)=\left(D_{z z}^{2} u\left(x, z_{0}\right)-D_{z z}^{2} v\left(y, z_{0}\right)\right)^{-1} D_{z y}^{2} v\left(y, z_{0}\right)
$$

which, substituted into (30) yields

$$
D_{x y}^{2} s(x, y)=D_{x z}^{2} u\left(x, z_{0}(x, y)\right)\left(D_{z z}^{2} u\left(x, z_{0}\right)-D_{z z}^{2} v\left(y, z_{0}\right)\right)^{-1} D_{z y}^{2} v\left(y, z_{0}\right) .
$$

By hypothesis, this matrix is positive definite as required.

When $n>1$, this lemma gives the setting whose empirical properties are studied in Heckman, Matzkin, and Nesheim [20]. When $n=1$, this reduces to the usual Spence-Mirrlees conditions $D_{x z}^{2} u \neq 0 \neq D_{y z}^{2} v$ on $u$ and $v$ separately plus strict concavity with respect to $z$ of the difference $u(x, z)-v(y, z)$.

\subsection{The subtwist: a weaker condition for uniqueness}

A priori, there does not seem to be any economic reason why the twist condition should be expected to hold. ${ }^{9}$ Whether or not twisting is necessary to guarantee purity of assignments for general measures $\mu_{0}$ and $\nu_{0}$ in the Lipschitz-buyer setting is an open question. The good news, however, is that it is certainly not necessary to guarantee uniqueness of the assignment $\gamma$. Though it is frequently assumed to be satisfied in applications where the spaces of buyers $X_{0}$ and sellers $Y_{0}$ are subsets of $\mathbf{R}^{n}$, this is not always the case. There are also important settings where twisting cannot be satisfied. Taking $X_{L}=\emptyset$ for simplicity, no differentiable surplus function satisfies the twist condition on a compact space $X_{0}$ such as the circle or sphere $\mathbf{S}^{n}:=\left\{\|x\|^{2}=1 \mid x \in \mathbf{R}^{n+1}\right\}$ - or the periodic cube $\mathbf{T}^{n}=\mathbf{R}^{n} / \mathbf{Z}^{n}$ - since $x \in X_{0} \longrightarrow s\left(x, y_{1}\right)-s\left(x, y_{2}\right)$ obviously has critical points where its maximum and minimum are attained. If the buyers

\footnotetext{
${ }^{9}$ For many common economic models, the twist condition cannot actually hold. For instance, it is typically violated in models of horizontal differentiation on a circle (see Example 4.20 for an illustration).
} 
and sellers were distributed continuously over the surface of the planet or around locations on an expressway encircling a city, there would be no hope of twisting. This situation is not much improved by assuming $X_{L}$ non-empty: no surplus $s(R x, R y)=s(x, y) \in C^{1}\left(\mathbf{S}^{n} \times \mathbf{S}^{n}\right)$ invariant under all rotations $R$ of the sphere $X_{0}=\mathbf{S}^{n}=Y_{0}$ can be twisted, since no negligible set $X_{L} \neq \emptyset$ is rotationally invariant. Similarly, no surplus $s(x+k, y+k)=s(x, y) \in C^{1}\left(\mathbf{T}^{n} \times \mathbf{T}^{n}\right)$ invariant under all translations $k \in \mathbf{R}^{n}$ can be twisted in the periodic setting. Clearly there are topological obstructions to twisting. It is a fundamental open question to understand when uniqueness of equilibria can be expected to persist in such settings. We give a sufficient condition which resolves this question in settings such as the circle and sphere - where $x \in X_{0} \longrightarrow s\left(x, y_{1}\right)-s\left(x, y_{2}\right)$ has only two critical points. This would not be the case in the periodic setting $\mathbf{T}^{2}$, and we do not know a single example of a smooth surplus function for which the (MK) solution to program (14) with $\mu_{0} \ll$ vol can be expected to be unique in this geometry.

The following theorem guarantees uniqueness of the optimal assignment $\gamma$. Even when all buyers elect to participate, there are many examples where the unique assignment will not be pure, meaning a positive fraction of buyers remain indifferent between two or more preferred sellers at equilibrium.

Definition 4.14 (Subtwist condition) In the Lipschitz-buyer setting, a surplus function $s: X \times Y \longrightarrow[0, \infty[$ is said to be subtwisted if there is a set $X_{L} \subset X_{0}$ of zero volume such that whenever $\partial^{x} s\left(x_{0}, y_{1}\right)$ intersects $\partial^{x} s\left(x_{0}, y_{2}\right)$ for some $x_{0} \in X_{0} \backslash X_{L}$ and $y_{1} \neq y_{2} \in Y$, then $x_{0}$ is either the unique global maximum or the unique global minimum of $s\left(x, y_{1}\right)-s\left(x, y_{2}\right)$ on $X=X_{0} \cup\left\{\emptyset_{X}\right\}$. The same definition applies in the semiconvex-buyer setting, except that $X_{L}$ must then lie in a $\mu$-negligible set, such as a (C $C^{2}$-rectifiable) hypersurface.

Example 4.15 A surplus differentiable with respect to $x$ is subtwisted if and only if there is a negligible set $X_{L} \subset X_{0}$ of buyers such that: for each distinct pair of sellers, $x \in X_{0} \backslash X_{L} \longrightarrow s\left(x, y_{1}\right)-s\left(x, y_{2}\right)$ has no critical points except for at most one global maximum and at most one global minimum.

Theorem 4.16 (Unique equilibria with mixed assignments) Let probability spaces $(X, \mu)$ and $(Y, \nu)$ and a surplus function $s(x, y)$ satisfy the subtwist condition in the Lipschitz-buyer or semiconvex-buyer setting. Then the maximizer $\gamma$ of (14) is unique. Moreover, $\gamma$ is supported on a numbered limb system with three limbs, as in Definition A.2.

Proof. Theorem 3.1 and Remark 3.2 provide a non-negative $s$-convex minimizing pair $(q, r)=\left(r^{s}, q^{\tilde{s}}\right)$ to the dual problem (16). Recalling that $\mu_{0} \ll v o l$ by hypothesis and that $q$ is locally Lipschitz [41], let Dom $D q \supset\left\{\emptyset_{X}\right\}$ denote the Borel subset of $X$ with full $\mu$-measure where $q$ is differentiable. Even in the semiconvex-buyer setting Dom $D q$ has full $\mu$-measure, because $q$ is locally semiconvex, hence differentiable except on a $C^{2}$ rectifiable hypersurface, to which $\mu_{0}$ assigns zero mass; (see footnote (1) of [16] and Theorem 10.26 of [41]). Taking 
$X_{L} \subset X_{0}$ as in the subtwist condition, let

$$
S:=\left\{(x, y) \in\left(\operatorname{Dom} D q \cap X \backslash X_{L}\right) \times Y \mid q(x)+r(y)-s(x, y)=0\right\}
$$

denote the set where the non-negative function $q(x)+r(y)-s(x, y)$ vanishes. Before embarking on the proof, recall any two equilibrium assignments $(x, y)$ and $\left(x^{\prime}, y^{\prime}\right)$ in $S$ satisfy

$$
s\left(x, y^{\prime}\right)+s\left(x^{\prime}, y\right) \leq s(x, y)+s\left(x^{\prime}, y^{\prime}\right) .
$$

This intuitive claim of Smith and Knott [36] dates partly back to Monge [30], and can be deduced by summing the inequalities

$$
\begin{array}{ll}
0 \leq q\left(x^{\prime}\right)+r(y)-s\left(x^{\prime}, y\right)= & q\left(x^{\prime}\right)+s(x, y)-q(x)-s\left(x, y^{\prime}\right) \\
0 \leq q(x)+r\left(y^{\prime}\right)-s\left(x, y^{\prime}\right)= & q(x)+s\left(x^{\prime}, y^{\prime}\right)-q\left(x^{\prime}\right)-s\left(x, y^{\prime}\right) .
\end{array}
$$

All measures $\gamma$ with left marginal $\mu$ assign full mass to $\left(X \cap \operatorname{Dom} D q \backslash X_{L}\right) \times Y$, since $X_{L}$ is $\mu$-negligible as a consequence of the subtwist hypotheses. Theorem 3.1 asserts that all optimizers $\gamma \in \Gamma(\mu, \nu)$ vanish outside $S$, and by upper semicontinuity of $s(x, y)$ that at least one optimal measure $\gamma$ exists. The proof will be complete if there exist maps $f_{3}: \operatorname{Dom} f_{3} \longrightarrow X_{0}$ and $f_{1}:$ Dom $f_{1} \longrightarrow\left\{\emptyset_{X}\right\}$ on disjoint subsets of $Y=\left(\operatorname{Dom} f_{1}\right) \cup\left(\operatorname{Dom} f_{3}\right)$ and $f_{2}: X_{0} \longrightarrow \operatorname{Dom} f_{1}$ such that $S \subset \operatorname{Antigraph}\left(f_{1}\right) \cup \operatorname{Graph}\left(f_{2}\right) \cup \operatorname{Antigraph}\left(f_{3}\right)$. Once this assertion is established, the results follow immediately from Theorem A.3 after identifying $I_{k}=\operatorname{Dom}\left(f_{k}\right)$ for $k=0,1,2,3, \operatorname{Dom} f_{2}=X_{0}$ and $\operatorname{Dom} f_{0}=\left\{\emptyset_{X}\right\}$.

A point $\left(x_{1}, y_{1}\right) \in S$ is said to be marked if $x_{1} \in X_{0}$ and

$$
s\left(x, y_{1}\right)-s\left(x, y_{2}\right) \geq s\left(x_{1}, y_{1}\right)-s\left(x_{1}, y_{2}\right)
$$

for all $x \in X$ and $\left(x_{1}, y_{2}\right) \in S$. Let $S_{1} \subset S$ denote the marked points in $S$, and $S_{2}=\left(X_{0} \times Y\right) \cap S \backslash S_{1}$ the unmarked points. We claim $\left(x_{1}, y_{1}\right) \in S_{2}$ and $\left(x_{2}, y_{1}\right) \in S$ forces $x_{1}=x_{2}$; in other words, the part of $S$ which projects to Dom $f_{3}:=\pi^{Y}\left(S_{2}\right)$ lies in an antigraph of unmarked points. The proof of this claim is inspired by the sole-supplier lemma [17]. Fix $\left(x_{1}, y_{1}\right) \in S_{2}$. Then $x_{1} \in X_{0} \cap \operatorname{Dom} D q \backslash X_{L}$ and $q(x)+r(y)-s(x, y) \geq 0$ is minimized at $\left(x_{1}, y_{1}\right)$, so the first order condition for a minimum implies $D q\left(x_{1}\right)=D_{x} s\left(x_{1}, y_{1}\right)$ if the latter exists, and $D q\left(x_{1}\right) \in \partial^{x} s\left(x_{1}, y_{1}\right)$ in any case. Since $\left(x_{1}, y_{1}\right)$ is unmarked, there exist some $\left(x_{1}, y_{2}\right) \in S$ and $x \in X$ which violate (35). Obviously, $D q\left(x_{1}\right) \in$ $\partial^{x} s\left(x_{1}, y_{2}\right)$ so the superdifferentials of $s$ intersect. The subtwist condition now guarantees $x_{1}$ is the unique maximizer of $s\left(\cdot, y_{1}\right)-s\left(\cdot, y_{2}\right)$; it cannot be the minimizer due to the presumed violation of (35). Any $\left(x_{2}, y_{1}\right) \in S$ therefore satisfies $s\left(x_{2}, y_{1}\right)-s\left(x_{2}, y_{2}\right)<s\left(x_{1}, y_{1}\right)-s\left(x_{1}, y_{2}\right)$, or else $x_{2}=x_{1}$. The strict inequality violates (34), establishing the claim $x_{1}=x_{2}$.

On Dom $f_{3}:=\pi^{Y}\left(S_{2}\right)$ define $f_{3}(y):=x$ for each $(x, y) \in S_{2}$. The preceding claim shows the part of $S$ which projects to Dom $f_{3}$ coincides precisely with $\operatorname{Antigraph}\left(f_{3}\right)=S_{2}$. We next show that both $\left(x_{1}, y_{1}\right)$ and $\left(x_{1}, y_{0}\right)$ in $S_{1}$ implies $y_{0}=y_{1}$, so $S_{1} \subset \operatorname{Graph}\left(f_{2}\right)$, where $f_{2}: X_{0} \longrightarrow Y$ is defined by

$$
f_{2}\left(x_{1}\right)= \begin{cases}y_{1} & \text { if }\left(x_{1}, y_{1}\right) \in S_{1} \\ \emptyset_{Y} & \text { if no }\left(x_{1}, y_{1}\right) \in S_{1} \text { exists. }\end{cases}
$$


Before addressing this claim, note our definition of $S_{2} \subset X_{0} \times Y$ ensures $\operatorname{Ran} f_{3} \subset$ Dom $f_{2}=X_{0}$. Also, as argued above, $\left(x_{1}, y_{1}\right) \in S \backslash S_{2}$ precludes $\left(x_{2}, y_{1}\right) \in S_{2}$, so (36) guarantees $\operatorname{Ran} f_{2} \subset \operatorname{Dom} f_{1}:=Y \backslash \operatorname{Dom} f_{3}$ after $\left(\emptyset_{X}, \emptyset_{Y}\right) \in S \backslash S_{2}$ is recalled. Finally, defining $f_{1}(y):=\emptyset_{X}$ ensures $\operatorname{Ran} f_{1} \subset \operatorname{Dom} f_{0}$, where Dom $f_{0}:=X \backslash X_{0}=\left\{\emptyset_{X}\right\}$. Also $\left(x_{1}, y_{1}\right) \in S \backslash\left(S_{1} \cup S_{2}\right)$ implies $x_{1}=\emptyset_{X}=f_{1}\left(y_{1}\right)$ while precluding $\left(x_{2}, y_{1}\right) \in S_{2}$, so $y_{1} \notin \operatorname{Dom} f_{3}$ and $S \backslash\left(S_{1} \cup S_{2}\right) \subset \operatorname{Antigraph}\left(f_{1}\right)$.

Since $S_{2}=\operatorname{Antigraph}\left(f_{3}\right)$ and $S_{1} \subset \operatorname{Graph}\left(f_{2}\right)$ we have verified all the hypotheses for the unique representation of Theorem A.3 to apply. It remains only to show $f_{2}\left(x_{1}\right)$ is well-defined by (36). To reach a contradiction, suppose $\left(x_{1}, y_{1}\right) \neq\left(x_{1}, y_{0}\right)$ both belong to $S_{1}$. According to (35), this means that $s\left(x, y_{1}\right)-s\left(x, y_{0}\right)$ and its negative $s\left(x, y_{0}\right)-s\left(x, y_{1}\right)$ are both minimized at $x=x_{1}$. But then $s\left(x, y_{1}\right)-s\left(x, y_{0}\right)=$ const independent of $x$, which violates the subtwist condition since then $\partial^{x} s\left(x, y_{0}\right)=\{\mathbf{0}\}=\partial^{x} s\left(x, y_{1}\right)$ for all $x \in X$. We conclude $f_{2}\left(x_{1}\right)$ is well-defined by (36) and the theorem established.

\subsection{Circular assignment: example of a subtwisted cost}

The preceding theorem generalizes results of Gangbo \& McCann [17] and Ahmad [1]. A special case of these earlier results yields the following illustrative example.

Example 4.17 (School districts on a ringroad) Consider a simple model of spatial matching in which a continuum of students and a continuum of schools are located at points on a circular expressway around a city. The pairwise surplus from matching a student to a school is a decreasing function of distance due to commuting costs; in particular, each student $x$ would prefer to be matched to a school with the same location as hers to minimize transportation expenses.

Formally, thus, let $X_{0}=Y_{0}=\mathbf{S}^{1}$ and $s(x, y)=1+\cos (2 \pi(x-y))$ where each $x$ represents a student and each $y$ represents a school. The rate at which the surplus decreases is increasing for $|x-y| \leq \frac{1}{4}$. However, it is decreasing for $\frac{1}{4} \leq|x-y| \leq \frac{1}{2}$. Note that $s(x, y) \geq 0$, so that participation is complete. Also, the model does not satisfy the twist condition. Indeed, the surplus $s$ is differentiable, but for any $\left(y_{1}, y_{2}\right)$ the function $s\left(x, y_{1}\right)-s\left(x, y_{2}\right)$ admits $x=$ $\frac{y_{1}+y_{2}}{2} \pm \frac{1}{4}$ as critical points.

Now, assume first that $\mu=\nu$, meaning students and schools have the same distribution on the circle. Then the unique solution of the primal surplus maximization problem would have support on the graph $y=x$. Every student would travel a maximum distance of zero. Any pair $(q, r)$ of non-negative constants $q(x)=q_{0}$ and $r(y)=r_{0}$ such that $q_{0}+r_{0}=2$ would solve the dual problem. This is a case in which the assignment is unique and pure, despite the fact that the twist condition does not apply. Note however, that the equilibrium price (22) is not uniquely determined until a choice of utility transferred $q_{0} \in[0,2]$ is made. This ambiguity in price would be resolved in scenarios where some students or schools choose not to participate, either because of a net imbalance between supply and demand, or due to a uniform increase in the commuting costs. 
However, the model becomes much more interesting when the densities associated with $\mu$ and $\nu$ are different. Assume, in particular, that they are as those given in Figure 1. Specifically, the distribution of students is concentrated around $x=\frac{1}{4}$ while the distribution of schools is concentrated around $y=\frac{3}{4}$. That is, most of the students live on the north side of the city while most of the schools are located on the south side. In this case, the optimal matching is still unique; but it is very different from the previous case. Indeed, it is impossible to match all students to a school near to their residence. The support of the unique optimal measure is shown in Figure 2. All students $x \in\left[0, \frac{1}{8}\right] \cup\left[\frac{3}{8}, 1\right]$, are matched to a single school near to their home. For example, $x=0.1$ is matched to $y=0.867$. All students $x \in\left(\frac{1}{8}, \frac{3}{8}\right)$ are matched to two schools; one at a distance less than or equal to $\frac{1}{4}$ and one at a distance greater than $\frac{1}{4}$. In the equilibrium these students are indifferent between the two locations. ${ }^{10}$ In each location, they obtain a surplus equal to $q(x)$. The surplus of the students and schools are displayed in Figure 3. The students and schools that are in scarce supply, $x=\frac{3}{4}$ and $y=\frac{1}{4}$, obtain the highest surplus. Those who are abundant, $x=\frac{1}{4}$ and $y=\frac{3}{4}$, obtain the lowest. The optimal measure assigns a fraction of each of the abundant students and schools to each of two locations, one less than a distance of $\frac{1}{4}$, one greater than this distance. Because there is such a large number of students near $x=\frac{1}{4}$ and schools near $y=\frac{3}{4}$, there is a social benefit from having some students travel a great distance. Technically, the measure $\gamma$ on each branch is calculated as follows. Figure 2 depicts two limbs of a numbered limb system:

$$
\begin{aligned}
& y=f_{2}(x) \text { for } x \in[0,1] \text { and } y \in\left(\frac{5}{8}, \frac{7}{8}\right) \\
& x=f_{3}(y) \text { for } y \in\left[0, \frac{5}{8}\right] \cup\left[\frac{7}{8}, 1\right] .
\end{aligned}
$$

The third limb $f_{1}(y):\left(\frac{5}{8}, \frac{7}{8}\right) \rightarrow\left\{\emptyset_{X}\right\}$ is not displayed. These limbs define the support of the optimal measure. The optimal measure is given by

$$
\begin{aligned}
\gamma_{3} & =\left.\left(f_{3} \times i d_{Y}\right)_{\#} \nu\right|_{\operatorname{Dom} f_{3}} \\
\gamma_{2} & =\left.\left(i d_{X} \times f_{2}\right)_{\#}\left(\mu-\pi_{\#}^{X} \gamma_{3}\right)\right|_{\operatorname{Dom} f_{2}} \\
\gamma_{1} & =\left.\left(f_{1} \times i d_{Y}\right)_{\#}\left(\nu-\pi_{\#}^{Y} \gamma_{2}\right)\right|_{\operatorname{Dom} f_{1}}=0 .
\end{aligned}
$$

In the example, we see the matches $(x, y) \in X \times Y_{0}$ between $\gamma$-a.e. participating pair can be found in the graph of one of two mappings $g:$ Dom $g \subset Y_{0} \longrightarrow X$ or $f: \operatorname{Dom} f \subset X_{0} \longrightarrow Y_{0}$, with range of $f$ disjoint from Dom $g$. This should be contrasted with the Spence-Mirrlees (twisted) case, where the matches lie on the graph of a single map $f: X_{0} \longrightarrow Y$, à la Monge. It can also be compared with the necessary and sufficient condition given in Corollary A.4 for a doubly stochastic measure $\gamma \in \Gamma(\lambda, \lambda)$ on the square $X_{0}=Y_{0}=[0,1]$ to be extremal,

\footnotetext{
${ }^{10}$ The apparent indifference of certain students to three or more schools is due to the limited resolution of our computation in Figure 2. Otherwise, the solution would violate a theorem of [17].
} 
which asserts that the support of $\gamma$ must lie in a numbered limb system, with at most countably many limbs, much as in Hestir and Williams [21]. In our theorem the system consists of two limbs, while in the twisted-buyer case it consists of a single limb. We do not know of any convenient condition on the surplus function which could lead to unique matches $\gamma$ concentrated on a system with three or more numbered limbs. However, developments so far suggest the maximal number of limbs must generally be linked to the complexity of the (Morse) critical point structure of the function $x \in X_{0} \longrightarrow s\left(x, y_{1}\right)-s\left(x, y_{2}\right)$.

Alternately, taking student assignments to schools to be fixed, Example 4.20 can parlayed into an example set on the periodic square $\mathbf{T}^{2}$ instead of the circle, by allowing students without cars to contract with students who drive to school to achieve desirable carpooling arrangements. There are then two kinds of students, and the type space of each is two-dimensional, consisting of a residential and a school location. In this case, topology forces even the subtwist condition to fail, leaving uniqueness an unresolved issue for all surplus functions!

\section{Multiple-agent contracts}

The hedonic pricing and matching problems we have discussed admit a natural generalization to the setting in which each contract $z$ requires the participation of $k$ agents chosen from different type spaces $\left(X_{1}, \mu_{1}\right), \ldots,\left(X_{k}, \mu_{k}\right)$ [9]. The case $k=2$ has been discussed above, but for $k>2$ we assume the utility of contract $z \in Z$ to agent $x \in X_{i}$ is given by an upper semicontinuous function $u_{i}: X_{i} \times Z \longrightarrow \mathbf{R} \cup\{-\infty\}$ plus any compensation $P_{i}(z)$ he receives. Thus the indirect utility available to him is

$$
U_{i}(x)=\sup _{z \in Z}\left\{u_{i}(x, z)+P_{i}(z)\right\}
$$

with the usual convention $-\infty+\infty=-\infty$. The payments $P_{i}(z)$ are Borel and assumed to satisfy a frictionless trading condition $0=\sum_{i=1}^{k} P_{i}(z)$ on $Z$ which prevents arbitrage and neglects friction. Payments corresponding to the null contract must vanish $P_{i}\left(\emptyset_{Z}\right)=0$. As before, each type space $X_{i}=X_{i}^{0} \cup\left\{\emptyset_{i}\right\}$ includes an isolated dummy agent type of mass

$$
\mu_{i}\left(\emptyset_{i}\right)=1+\sum_{j \neq i} \mu_{j}\left(X_{j}^{0}\right)
$$

and satisfies

$$
u_{i}(x, z)=\left\{\begin{array}{cl}
0 & \text { if } z=\emptyset_{Z} \text { and } x \in X_{i}, \\
-\infty & \text { if } z \in Z \backslash\left\{\emptyset_{Z}\right\} \text { and } x=\emptyset_{i} .
\end{array}\right.
$$

A joint measure $\alpha$ on $X_{1} \times \cdots \times X_{k} \times Z$ together with frictionless payment schedules $P_{i}: Z \longrightarrow \mathbf{R} \cup\{ \pm \infty\}$ represent a market clearing equilibrium if it has marginals $\pi_{\#}^{X_{i}} \alpha=\mu_{i}$ for each $i=1, \ldots, k$, and

$$
U_{i}\left(x_{i}\right)=u_{i}\left(x_{i}, z\right)+P_{i}(z)
$$


holds for each $i=1, \ldots, k$ and $\alpha$-a.e. $\left(x_{1}, \ldots, x_{k}, z\right) \in \operatorname{Spt} \alpha$.

Define the non-negative surplus function

$$
s\left(x_{1}, \ldots, x_{k}\right)=\max _{z \in Z} \sum_{i=1}^{k} u_{i}\left(x_{i}, z\right) .
$$

The same arguments presented above show equivalence of this hedonic pricing problem to the linear program

$$
\max _{\gamma} \gamma[s]
$$

where the maximum is taken over all joint measures $\gamma \geq 0$ on $X_{1} \times \cdots \times X_{k}$ having prescribed marginals $\mu_{i}=\pi_{\#}^{X_{i}} \gamma$. The dual infimum

$$
\min _{q_{i}: X_{i} \longrightarrow[0, \infty]} \sum_{i=1}^{k} \mu_{i}\left[q_{i}\right]
$$

is taken over functions $q_{i}$ satisfying $s\left(x_{1}, \ldots, x_{k}\right) \leq \sum_{i=1}^{k} q_{i}\left(x_{i}\right)$ on $X_{1} \times \cdots \times X_{k}$, normalized so $q_{1}\left(\emptyset_{1}\right)=0$. Duality still holds $\gamma[s] \leq \sum \mu_{i}\left[q_{i}\right]$, with equality if and only if $\gamma$ is a maximizer and $\left(q_{1}, \ldots, q_{k}\right)$ minimizes. As before, it follows from $q_{1}\left(\emptyset_{1}\right)=0$ that $q_{i}\left(\emptyset_{i}\right)=0$ for each $i \leq k$. The existence and characterization of maximizers, minimizers, and equilibria is identical, but the literature exploring conditions on the surplus which guarantee uniqueness of the maximizing assignment or assortative matching is much more limited in the multiple marginal case; see [33] [15] for references. Let us only observe that the uniqueness of marginal payoffs proved in Proposition 4.8 extends immediately to the multiple agent problem. Thus if $\mu_{1}$ vanishes on all $\left(C^{2}\right.$-rectifiable) hypersurfaces of a smooth manifold $X_{1}^{0}:=X_{1} \backslash\left\{\emptyset_{1}\right\}$, and the surplus function $s \in C\left(X_{1} \times \ldots X_{k}\right)$ is semiconvex on $X_{1}^{0}$, uniformly in the other $k-1$ variables, and $\left(q_{1}, \ldots, q_{k}\right)$ minimizes (38) with $q_{1}$ semiconvex, then $D q_{1}$ is uniquely determined $\mu_{1}$ almost everywhere on $X_{1}^{0}$.

\section{A Supports of extremal doubly stochastic mea- sures}

A characterization of extremal doubly stochastic measures in terms of their supports has been sought [5, Problem 111] since Birkhoff and von-Neumann solved the analogous finite dimensional problem by characterizing extremal doubly stochastic matrices as permutations [4] [42]. Significant progress was made by Beneš and Stĕpán, who gave a condition on the support of a doubly stochastic measure necessary for the measure to be extremal [3]. Hestir and Williams refined this condition, showing that it becomes sufficient under an additional Borel measurability hypothesis which unfortunately, is not always satisfied [21]. In this appendix we complete this line of research by closing the gap between the preceding results, showing just enough measurability is built into the problem to derive a simultaneously necessary and sufficient condition on the support 
of a doubly stochastic measure to guarantee extremality. Our improvement is achieved using Villani's argument from Theorem 5.28 [41].

An important property of pure solutions is that the equilibrium is uniquely determined by the profile of buyers and their strategies. Specifically, the following technical result improves Lemma 2.4 of [17], allowing us to establish a unique representation of the equilibrium measure simply by showing that almost all sellers have pure preferences at equilibrium. The same lemma will also be used to remove the measurability hypothesis which distinguishes Hestir and Williams' necessary condition from their sufficient condition for a doubly stochastic measure on the square to be extremal [21]. Indeed, from their work it follows that although pure measures are extremal in $\Gamma(\mu, \nu)$, the converse is far from being true; this peculiarity is an inevitable feature of continuous type spaces $X$.

Lemma A.1 (Pure measures are push-forwards) Let $X_{0}$ and $Y_{0}$ be Borel subsets of complete separable metric spaces, and $\gamma \geq 0$ a $\sigma$-finite Borel measure on the product space $X_{0} \times Y_{0}$. Denote the left marginal of $\gamma$ by $\mu_{0}:=\pi_{\#}^{X_{0}} \gamma$. If $\gamma$ is concentrated on the graph of $f: X_{0} \longrightarrow Y_{0}$, meaning $\left\{(x, y) \in X_{0} \times Y_{0} \mid y \neq\right.$ $f(x)\}$ has zero outer measure, then $f$ is $\mu_{0}$-measurable and $\gamma=\left(i d_{X_{0}} \times f\right)_{\#} \mu_{0}$.

Proof. Since outer-measure is subadditive, it costs no generality to assume the Borel subsets $X_{0}$ and $Y_{0}$ are in fact complete and separable. Any $\sigma$-finite Borel measure $\gamma$ is regular and $\sigma$-compact on a complete separable metric space; e.g. p. 255 of [11] or Theorem I-55 of [40]. Since $\gamma$ vanishes outside $\operatorname{Graph}(f):=$ $\left\{(x, f(x)) \mid x \in X_{0}\right\}$, there is an increasing sequence of compact sets $K_{i} \subset$ $K_{i+1} \subset \operatorname{Graph}(f)$ whose union $K_{\infty}=\lim _{i \rightarrow \infty} K_{i}$ contains the full mass of $\gamma$. Compactness of $K_{i} \subset \operatorname{graph}(f)$ implies continuity of $f$ on the compact projection $X_{i}:=\pi^{X}\left(K_{i}\right)$. Thus the restriction $f_{\infty}$ of $f$ to $X_{\infty}:=\pi^{X}\left(K_{\infty}\right)$ is a Borel map whose graph $K_{\infty}=\operatorname{Graph}\left(f_{\infty}\right)$ is a $\sigma$-compact set of full measure for $\gamma$. We now verify that $\gamma$ and $\left(i d_{X_{\infty}} \times f_{\infty}\right)_{\#} \mu_{0}$ assign the same mass to each Borel rectangle $U \times V \subset X_{0} \times Y_{0}$. Since $(U \times V) \cap \operatorname{Graph}\left(f_{\infty}\right)=\left(\left(U \cap f_{\infty}^{-1}(V)\right) \times\right.$ $\left.Y_{0}\right) \cap \operatorname{Graph}\left(f_{\infty}\right)$ we find

$$
\begin{aligned}
\gamma(U \times V) & =\gamma\left(\left(U \cap f_{\infty}^{-1}(V)\right) \times Y_{0}\right) \\
& =\mu_{0}\left(U \cap f_{\infty}^{-1}(V)\right),
\end{aligned}
$$

proving $\gamma=\left(i d_{X_{\infty}} \times f_{\infty}\right)_{\#} \mu_{0}$. Taking $U=X_{0} \backslash X_{\infty}$ and $V=Y_{0}$ shows $X_{0} \backslash X_{\infty}$ is $\mu_{0}$-negligible. Since $i d_{X_{0}} \times f$ differs from the Borel map $i d_{X_{\infty}} \times f_{\infty}$ only on the $\mu_{0}$-negligible complement of the $\sigma$-compact set $X_{\infty}$, we conclude $f$ is $\mu_{0}$-measurable and $\gamma=\left(i d_{X_{0}} \times f\right)_{\#} \mu_{0}$ as desired.

The preceding lemma shows that any measure is concentrated on a graph is uniquely determined by its marginals. This would be the case for optimal measures in the twisted-buyer setting. Uniqueness, however, does not require the twist condition to be satisfied; as the next result demonstrates, the sufficient condition given by Theorem 4.11 is far from necessary for uniqueness of the equilibrium. 
Given a map $f: D \longrightarrow Y$ on $D \subset X$, we denote its graph, domain, range, and the graph of its (multivalued) inverse by

$$
\begin{aligned}
& \operatorname{Graph}(f):=\quad\{(x, f(x)) \mid \quad x \in D\}, \\
& \operatorname{Dom} f:=\quad \pi^{X}(\operatorname{Graph}(f)) \quad=D, \\
& \operatorname{Ran} f:=\quad \pi^{Y}(\operatorname{Graph}(f)), \\
& \operatorname{Antigraph}(f):=\{(f(x), x) \mid x \in \operatorname{Dom} f\} \quad \subset Y \times X \text {. }
\end{aligned}
$$

More typically, we will be interested in the $\operatorname{Antigraph}(g) \subset X \times Y$ of a map $g: D \subset Y \longrightarrow X$. Following Hestir and Williams [21] we define:

Definition A.2 (Numbered limb system) Let $X_{0}$ and $Y_{0}$ be Borel subsets of complete separable metric spaces. A relation $S \subset X_{0} \times Y_{0}$ is a numbered limb system if there is a countable disjoint decomposition of $X_{0}=\cup_{i=0}^{\infty} I_{2 i+1}$ and of $Y_{0}=\cup_{i=0}^{\infty} I_{2 i}$ with a sequence of maps $f_{2 i}: \operatorname{Dom}\left(f_{2 i}\right) \subset X_{0} \longrightarrow Y_{0}$ and $f_{2 i+1}$ : $\operatorname{Dom}\left(f_{2 i+1}\right) \subset Y_{0} \longrightarrow X_{0}$ such that $S=\cup_{i=1}^{\infty} \operatorname{Graph}\left(f_{2 i}\right) \cup \operatorname{Antigraph}\left(f_{2 i-1}\right)$, with $\operatorname{Dom}\left(f_{k}\right) \cup \operatorname{Ran}\left(f_{k+1}\right) \subset I_{k}$ for each $k \geq 0$. The system has (at most) $N$ limbs if $\operatorname{Dom}\left(f_{k}\right)=\emptyset$ for all $k>N$.

Notice the map $f_{0}$ is irrelevant to this definition; we may always take $\operatorname{Dom}\left(f_{0}\right)=$ $\emptyset$, but require $\operatorname{Ran}\left(f_{1}\right) \subset I_{0}$. The point is the following theorem and its corollary, which improves the results proved by Hestir and Williams for Lebesgue measure $\mu_{0}=\nu_{0}=\lambda$ on the interval $X_{0}=Y_{0}=[0,1]$, as well as extending them to other spaces.

Theorem A.3 (Numbered limb systems support unique equilibria) Let $X_{0}$ and $Y_{0}$ be Borel subsets of complete separable metric spaces, and $\gamma \geq 0$ a $\sigma$-finite Borel measure on the product space $X_{0} \times Y_{0}$. Denote the marginal projections of $\gamma$ by $\mu:=\pi_{\#}^{X_{0}} \gamma$ and $\nu:=\pi_{\#}^{Y_{0}} \gamma$. Suppose there is a numbered limb system $S=\cup_{i=1}^{\infty} \operatorname{Graph}\left(f_{2 i}\right) \cup \operatorname{Antigraph}\left(f_{2 i-1}\right)$ whose complement has zero outer measure for $\gamma$. If the system has finitely many limbs or $\gamma\left[X_{0} \times Y_{0}\right]<\infty$, then $\gamma$ is uniquely determined by $S, \mu$ and $\nu$. In fact, $\gamma=\sum_{k=1}^{\infty} \gamma_{k}$ where

$$
\begin{aligned}
\gamma_{2 i}=\left(i d_{X_{0}} \times f_{2 i}\right)_{\#} \eta_{2 i}, & \gamma_{2 i-1} & =\left(f_{2 i-1} \times i d_{Y_{0}}\right)_{\#} \eta_{2 i-1}, \\
\eta_{2 i}=\left.\left(\mu-\pi_{\#}^{X_{0}} \gamma_{2 i+1}\right)\right|_{\text {Dom } f_{2 i}}, & \eta_{2 i-1} & =\left.\left(\nu-\pi_{\#}^{Y_{0}} \gamma_{2 i}\right)\right|_{\text {Dom } f_{2 i-1}} .
\end{aligned}
$$

Here $f_{k}$ is measurable with respect to the $\eta_{k}$ completion of the Borel $\sigma$-algebra. If the system has $N<\infty$ limbs, $\gamma_{k}=0$ for $k>N$, and $\eta_{k}$ and $\gamma_{k}$ can be computed recursively from the formulae above starting from $k=N$.

Proof. Let $S=\cup_{i=1}^{\infty} \operatorname{Graph}\left(f_{2 i}\right) \cup \operatorname{Antigraph}\left(f_{2 i-1}\right)$ be a limb numbered tree system whose complement has zero outer measure for $\gamma$. This means that $I_{k}=$ Dom $f_{k}$ gives a disjoint decomposition of $X_{0}=\cup_{i=0}^{\infty} I_{2 i}$ and of $Y_{0}=\cup_{i=0}^{\infty} I_{2 i+1}$, and that $\operatorname{Ran}\left(f_{k}\right) \subset \operatorname{Dom}\left(f_{k-1}\right)$ for each $k \geq 1$. The graphs $\operatorname{Graph}\left(f_{2 i}\right)$ are disjoint since their domains $I_{2 i}$ are disjoint, and the antigraphs Antigraph $\left(f_{2 i-1}\right)$ are disjoint since their domains $I_{2 i-1}$ are. Moreover, $\operatorname{Graph}\left(f_{2 i}\right)$ is disjoint from 
$\operatorname{Antigraph}\left(f_{2 j-1}\right)$ for all $i, j \geq 1: \operatorname{Ran}\left(f_{2 i}\right) \subset \operatorname{Dom}\left(f_{2 i-1}\right)$ prevents $\operatorname{Graph}\left(f_{2 i}\right)$ from intersecting $\operatorname{Antigraph}\left(f_{2 j-1}\right)$ unless $j=i$ since the domains $I_{2 j-1}$ are disjoint, and Graph $\left(f_{2 i}\right)$ cannot intersect Antigraph $\left(f_{2 i-1}\right)$ since $\operatorname{Dom}\left(f_{2 i}\right)$ is disjoint from $\operatorname{Ran}\left(f_{2 i-1}\right) \subset \operatorname{Dom}\left(f_{2 i-2}\right)$. As in the preceding lemma, the Borel regularity of $\gamma$ implies the existence of compact sets $K_{k}^{j} \subset\left(\right.$ Anti) $\operatorname{Graph}\left(f_{k}\right)$ such that the outer measure $\gamma\left((\right.$ Anti $\left.) \operatorname{Graph}\left(f_{k}\right) \backslash K_{k}^{j}\right)<2^{-k-j}$. Here (Anti) $\operatorname{Graph}\left(f_{k}\right)$ denotes a graph if $k$ is even and an antigraph if $k$ is odd. Setting $K_{k}^{\infty}=\cup_{j=1}^{\infty} K_{k}^{j}$ yields a collection of $\sigma$-compact sets whose union $K^{\infty}=\cup_{k=1}^{\infty} K_{k}^{\infty}$ exhausts the measure of $\gamma$. Letting $\gamma_{k}=\left.\gamma\right|_{K_{k}^{\infty}}$, denote the restriction of $\gamma$ to $K_{k}^{\infty}$, disjointness of $K_{k}^{\infty} \subset(\operatorname{Anti}) \operatorname{Graph}\left(f_{k}\right)$ yields $\gamma=\sum_{k=1}^{\infty} \gamma_{k}$. Defining the marginal projections $\mu_{k}=\pi_{\#}^{X_{0}} \gamma_{k}, \nu_{k}=\pi_{\#}^{Y_{0}} \gamma_{k}, \eta_{2 i}=\mu_{2 i}$ and $\eta_{2 i-1}=\mu_{2 i-1}$, we recover (39) and the $\eta_{k}$-measurability of $f_{k}$ immediately from Lemma A.1. Since $\mu_{2 i}$ vanishes outside Dom $f_{2 i}$, we see $\mu_{2 i}=\left.\left(\mu-\sum_{k \neq 2 i} \mu_{k}\right)\right|_{\text {Dom } f_{2 i}}$. For $k$ even, $\mu_{k}$ vanishes outside $\operatorname{Dom}\left(f_{k}\right)$, while for $k$ odd, $\mu_{k}$ vanishes outside $\operatorname{Ran} f_{k} \subset \operatorname{Dom}\left(f_{k-1}\right)$, which is disjoint from Dom $f_{2 i}$ unless $k=2 i+1$. Thus $\eta_{2 i}=\left.\left(\mu-\mu_{2 i+1}\right)\right|_{\operatorname{Dom} f_{2 i}}$. The formula (40) for $\eta_{2 i-1}$ follows from similar considerations.

It remains to show the representation (39)-(40) specifies $\left(\gamma_{k}, \eta_{k}\right)$ uniquely for all $k \geq 1$, and hence determines $\gamma=\sum \gamma_{k}$ uniquely. If the system has $N<\infty$ limbs, $I_{k}=\emptyset$ for $k>N$ and hence $\gamma_{k}=0$. We can compute $\eta_{k}$ and $\gamma_{k}$ starting with $k=N$, and then recursively from the formulae above for $k=N-1, N-$ $2, \ldots, 1$, so the formulae represent $\gamma$ uniquely. If instead $S$ has countably many limbs, suppose there are two finite Borel measures $\gamma$ and $\bar{\gamma}$ concentrated on $S$ with the same marginals $\mu$ and $\nu$. Expand the compact sets $K_{k}^{j}$ if necessary, to ensures the union $K_{k}^{\infty}$ exhausts both $\gamma$ and $\bar{\gamma}$ on (Anti)Graph $\left(f_{k}\right)$. Given $\epsilon>0$, take $N$ large enough so that both $\gamma$ and $\bar{\gamma}$ assign mass less than $\epsilon$ to $\cup_{k=N}^{\infty} K_{k}^{\infty}$. Setting $\gamma_{k}=\left.\gamma\right|_{K_{k}}$ and $\bar{\gamma}_{k}=\left.\bar{\gamma}\right|_{K_{k}}$, we find both $\gamma^{\epsilon}:=\sum_{k=1}^{N} \gamma_{k}$ and $\bar{\gamma}^{\epsilon}:=\sum_{k=1}^{N} \bar{\gamma}_{k}$ are concentrated on the same numbered limb system; it has finitely many limbs, and the differences $\delta \mu^{\epsilon}=\pi_{\#}^{X_{0}}\left(\bar{\gamma}^{\epsilon}-\gamma^{\epsilon}\right)$ and $\delta \nu^{\epsilon}=\bar{\nu}^{\epsilon}-\nu^{\epsilon}$ between their marginals have total variation at most $2 \epsilon$. Since the restrictions $\delta \mu_{2 i}^{\epsilon}=\left.\delta \mu^{\epsilon}\right|_{\operatorname{Dom} f_{2 i}}$ are disjoint, as are $\delta \nu_{2 i-1}^{\epsilon}=\left.\delta \nu^{\epsilon}\right|_{\operatorname{Dom} f_{2 i-1}}$, we find the sum of the total variations of

$$
\delta \eta_{k}^{\epsilon}:=\left\{\begin{array}{cc}
\bar{\mu}_{k}^{\epsilon}-\mu_{k}^{\epsilon} & k \text { even } \\
\bar{\nu}_{k}^{\epsilon}-\nu_{k}^{\epsilon} & k \text { odd }
\end{array}\right.
$$

is bounded: $\sum_{k=1}^{N}\left\|\delta \eta_{k}\right\|_{T V\left(\operatorname{Dom} f_{k}\right)}<4 \epsilon$. Using (39) to derive

$$
\begin{aligned}
\left\|\bar{\gamma}_{k}^{\epsilon}-\gamma_{k}^{\epsilon}\right\|_{T V\left(X_{0} \times Y_{0}\right)} & = \begin{cases}\left\|\left(i d_{X_{0}} \times f_{k}\right)_{\#} \delta \eta_{k}\right\|_{T V\left(X_{0} \times Y_{0}\right)} & k \text { even, } \\
\left\|\left(f_{k} \times i d_{Y_{0}}\right)_{\#} \delta \eta_{k}\right\|_{T V\left(X_{0} \times Y_{0}\right)} & k \text { odd },\end{cases} \\
& =\left\|\delta \eta_{k}\right\|_{T V\left(\operatorname{Dom} f_{k}\right)}
\end{aligned}
$$

and summing on $k$ yields $\left\|\bar{\gamma}^{\epsilon}-\gamma^{\epsilon}\right\|_{T V\left(X_{0} \times Y_{0}\right)}<4 \epsilon$. Since $\gamma^{\epsilon} \rightarrow \gamma$ and $\bar{\gamma}^{\epsilon} \rightarrow \bar{\gamma}$ as $\epsilon \rightarrow 0$, we conclude $\bar{\gamma}=\gamma$ to complete the uniqueness proof.

Corollary A.4 (Characterizing extremal doubly stochastic measures) Fix $X_{0}=Y_{0}=[0,1]$ and a Borel probability measure $\gamma \geq 0$ on the square 
$[0,1]^{2}$ whose marginal projections both coincide with Lebesgue measure $\pi_{\#}^{X_{0}} \gamma=$ $\lambda=\pi_{\#}^{Y_{0}} \gamma$. Then $\gamma$ is extremal in the convex set $\Gamma(\lambda, \lambda)$ of probability measures with the same marginals, if and only if there is a numbered limb system $S=\cup_{i=1}^{\infty} \operatorname{Graph}\left(f_{2 i}\right) \cup \operatorname{Antigraph}\left(f_{2 i-1}\right) \subset[0,1]^{2}$ whose complement has zero outer measure for $\gamma$.

Proof. First suppose $\gamma \in \Gamma(\lambda, \lambda)$ is an extreme point, meaning it cannot be expressed as a convex combination $\gamma=(1-t) \gamma_{0}+t \gamma_{1}$ of measures $\gamma_{0}, \gamma_{1} \in$ $\Gamma(\lambda, \lambda)$ with $0<t<1$ unless $\gamma_{0}=\gamma_{1}$. In this case Hestir and Williams [21] deduced the existence of a numbered limb system $S \subset X_{0} \times Y_{0}$ whose complement has zero outer measure for $\gamma$ from the work of Douglas [10] and Lindenstrauss [26].

To prove the converse, we shall suppose such a numbered limb system exists and deduce the extremality of $\gamma$. Let $\gamma=(1-t) \gamma_{0}+t \gamma_{1}$ with $\gamma_{0}, \gamma_{1} \in \Gamma(\mu, \nu)$ and $0<t<1$. Then $\gamma \geq \gamma_{0}$ and $\gamma \geq \gamma_{1}$, so $\gamma_{0}$ and $\gamma_{1}$ both assign zero outer measure to $S$. According to Theorem A.3, they are uniquely determined by $S$ and their marginals, hence $\gamma_{0}=\gamma_{1}$ and the corollary is proved.

Standard measure-space isomorphism results extend the support characterization of extremality, given by this corollary, to probability measures with fixed marginals $\mu$ and $\nu$ on Borel subsets of complete separable metric spaces.

\section{B Computation}

We use Example 4.17 to illustrate our computational algorithm for approximating solutions to the optimal transportation problem. In this example the surplus function is

$$
s(x, y)=1+\cos (2 \pi|x-y|)
$$

and the densities of $x$ and $y$ are depicted in Figure 1. The densities of $x$ and $y$ are periodic, bounded away from zero and symmetric with modes at $\frac{1}{4}$ and $\frac{3}{4}$ respectively.

To discretize the problem, we search for solutions to the dual in a space of periodic cubic spline functions. ${ }^{11}$ Define

$$
\mathbf{S}_{n 3}=\left\{f(x) \mid f(x)=\sum_{j=1}^{n+2} a_{j} S_{j}(x) \text { for } a \in A:\right\}
$$

where $S_{j}(x)$ is the $j$ 'th $B$-spline of degree three and

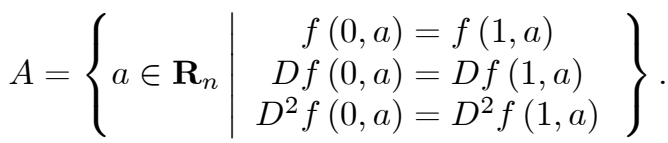

\footnotetext{
${ }^{11} \mathrm{We}$ also used Fourier series. In this example, the spline basis functions produced more stable and more accurate approximations for a given number of terms in the approximation.
} 
Then $\mathbf{S}_{n} \subseteq C_{2}[0,1]$ is the space of periodic cubic spline functions with $n$ knots in $[0,1]$. The set $A$ is a linear subspace of $\mathbf{R}_{n}$ and $\mathbf{S}_{n}$ is a linear subspace of the standard spline space defined on $[0,1] .{ }^{12}$

Rather than solve the linear program directly, we solve the following:

$$
\min _{q, r \in \mathbf{S}_{n}} \int q(x, a) d \mu+\int r(y, b) d \nu
$$

subject to

$$
\begin{aligned}
& q(x, a) \geq \max _{y \in Y} s(x, y)-r(y, b) \text { for } x \in\left\{x_{1}, \ldots, x_{n}\right\} \\
& r(y, a) \geq \max _{x \in X} s(x, y)-q(x, a) \text { for } y \in\left\{y_{1}, \ldots, y_{n}\right\} .
\end{aligned}
$$

This method has several benefits. First, the resulting solutions are nearly $s$ convex and $\tilde{s}$-convex. Second, the discretization has $2 n$ nonlinear constraints rather than the $n^{2}$ linear constraints that would result from a pointwise discretization of the dual linear program constraints. Moreover, the algorithm concentrates computational effort on regions of $X \times Y$ where the constraints are binding, that is near the zero set

$$
S=\{(x, y) \mid q(x, a)+r(y, b)=s(x, y)\} .
$$

Finally, the algorithm produces an estimate of the support of the measure $\gamma$ that maximizes the primal problem. For each $x$, this set valued map is

$$
y=m(x)=\arg \max _{\bar{y} \in Y} s(x, \bar{y})-r(\bar{y}, b) .
$$

The approximate solutions depicted in Figures 3 and 2 were computed $n=7$ equally spaced knots on the interval $[0,1]$.

\section{References}

[1] N. Ahmad. The geometry of shape recognition via a Monge-Kantorovich optimal transport problem. PhD thesis, Brown University, 2004.

[2] E.J. Anderson and P. Nash. Linear Programming in Infinite-Dimensional Spaces. Wiley, Chichester, 1987.

[3] V. Beneš and J. Stěpán. The support of extremal probability measures with given marginals. In M.L. Puri, P. Révész and W. Wertz, editors, Mathematical Statistics and Probability Theory, volume A. Theoretical Aspects, pages 33-41. D. Reidel Publishing Co., Dordrecht, 1987.

[4] G. Birkhoff. Tres observaciones sobre el algebra lineal. Univ. Nac. Tucumán. Revista A 5 (1946) 147-150.

${ }^{12}$ See for example Nürnberger [31]. 
[5] G. Birkhoff. Lattice Theory (revised edition). American Mathematical Society, New York, 1948.

[6] Y. Brenier. The dual least action problem for an ideal, incompressible fluid. Arch. Rational Mech. Anal. 122 (1993) 323-351.

[7] L.A. Caffarelli and R.J. McCann. Free boundaries in optimal transport and Monge-Ampère obstacle problems. To appear in Ann. of Math. (2).

[8] G. Carlier. Duality and existence for a class of mass transportation problems and economic applications. Adv. Math. Econom. 5 (2003) 1-21.

[9] G. Carlier and I. Ekeland. In preparation.

[10] R.D. Douglas. On extremal measures and subspace density. Michigan Math. J. 11 (1964) 243-246.

[11] R.M. Dudley. Real Analysis and Probability. Revised reprint of the 1989 original. Cambridge University Press, Cambridge, 2002.

[12] I. Ekeland. An optimal matching problem. Control Optim. Calc. Var. 11 1 (2005) 57-71.

[13] I. Ekeland. Existence, uniqueness and efficiency of equilibrium in hedonic markets with multidimensional types. Preprint at www.math.ubc.ca/ ekeland, 2005.

[14] W. Gangbo. Habilitation thesis. Université de Metz, 1995.

[15] W. Gangbo and A. Święch. Optimal maps for the multidimensional MongeKantorovich problem. Comm. Pure Appl. Math. 51 (1998) 23-45.

[16] W. Gangbo and R.J. McCann. The geometry of optimal transportation. Acta Math. 177 (1996) 113-161.

[17] W. Gangbo and R.J. McCann. Shape recognition via Wasserstein distance. Quart. Appl. Math. 58 (2000) 705-737.

[18] N.E. Gretsky, J.M. Ostroy, and W.R. Zame. The nonatomic assignment model. Econom. Theory 2 (1992) 103-127.

[19] N.E. Gretsky, J.M. Ostroy, and W.R. Zame. Perfect competition in the continuous assignment model. J. Econom. Theory 88 (1999) 60-118.

[20] J.J. Heckman, R. Matzkin, and L.P. Nesheim. Nonparametric estimation of nonadditive hedonic models. CEMMAP Working Paper CWP03/05, 2005.

[21] K. Hestir and S.C. Williams. Supports of doubly stochastic measures. Bernoulli 1 (1995) 217-243.

[22] W. Hildenbrand. Core and Equilibria of a Large Economy. Princeton University Press, Princeton, 1974. 
[23] L. Kantorovich. On the translocation of masses. C.R. (Doklady) Acad. Sci. URSS (N.S.) 37 (1942) 199-201.

[24] H.G. Kellerer. Duality theorems for marginal problems. Z. Wahrsch. Verw. Gebiete 67 (1984) 399-432.

[25] Y.-H. Kim and R.J. McCann. Continuity, curvature, and the general covariance of optimal transportation. Preprint at www.math.toronto.edu/mccann.

[26] J. Lindenstrauss. A remark on doubly stochastic measures. Amer. Math. Monthly 72 (1965) 379-382.

[27] X.-N. Ma, N. Trudinger and X.-J. Wang. Regularity of potential functions of the optimal transportation problem. Arch. Rational Mech. Anal. 177 (2005) 151-183.

[28] R.J. McCann. Existence and uniqueness of monotone measure-preserving maps. Duke Math. J. 80 (1995) 309-323.

[29] R.J. McCann. Exact solutions to the transportation problem on the line. R. Soc. Lond. Proc. Ser. A Math. Phys. Eng. Sci., 455 (1999) 1341-1380.

[30] G. Monge. Mémoire sur la théorie des déblais et de remblais. Histoire de l'Académie Royale des Sciences de Paris, avec les Mémoires de Mathématique et de Physique pour la même année, pages 666-704, 1781.

[31] Günther Nürnberger. Approximation by Spline Functions. Springer-Verlag, Berlin, 1980.

[32] A.Yu. Plakhov. Exact solutions of the one-dimensional Monge-Kantorovich problem (Russian). Mat. Sb. 195 (2004) 57-74.

[33] S.T. Rachev and L. Rüschendorf. Mass Transportation Problems. Probab. Appl. Springer-Verlag, New York, 1998.

[34] A. Roth and M. Sotomayor. Two-sided Matching: A study in Gametheoretic Modeling and Analysis. Econometric Society Monograph Series. Cambridge University Press, Cambridge, 1990.

[35] L.S. Shapley and M. Shubik. The assignment game I: the core. International Journal of Game Theory bf 1 (1972) 111-130.

[36] C. Smith and M. Knott. On Hoeffding-Fréchet bounds and cyclic monotone relations. J. Multivariate Anal. 40 (1992) 328-334.

[37] L. Uckelmann. Optimal couplings between onedimensional distributions. In V. Beneš and J. Stěpán, editors, Distributions with given marginals and moment problems, pages 261-273. Kluwer Academic Publishers, Dordrecht, 1997. 
[38] L. Veselý and L. Zajíček. Delta-convex mappings between Banach spaces and applications. Dissertationes Math. (Rozprawy Mat.) 289 (1989) 1-52.

[39] C. Villani. Topics in Optimal Transportation, volume 58 of Graduate Studies in Mathematics. American Mathematical Society, Providence, 2003.

[40] C. Villani. Cours d'Intégration et Analyse de Fourier. Preprint at http://www.umpa.ens-lyon.fr/ cvillani/Cours/iaf-2006.html, 2006.

[41] C. Villani. Optimal Transport, Old and New. St. Flour Lecture Notes. Springer, New York, to appear. Preprint at http://www.umpa.enslyon.fr/ cvillani/surveys.html\#oldnew.

[42] J. von Neumann. A certain zero-sum two-person game equivalent to the optimal assignment problem. In Contributions to the theory of games, volume 2, pages 5-12. Princeton University Press, Princeton NJ, 1953. 
Figure 1: Densities of students and schools on the circle in Example 4.17
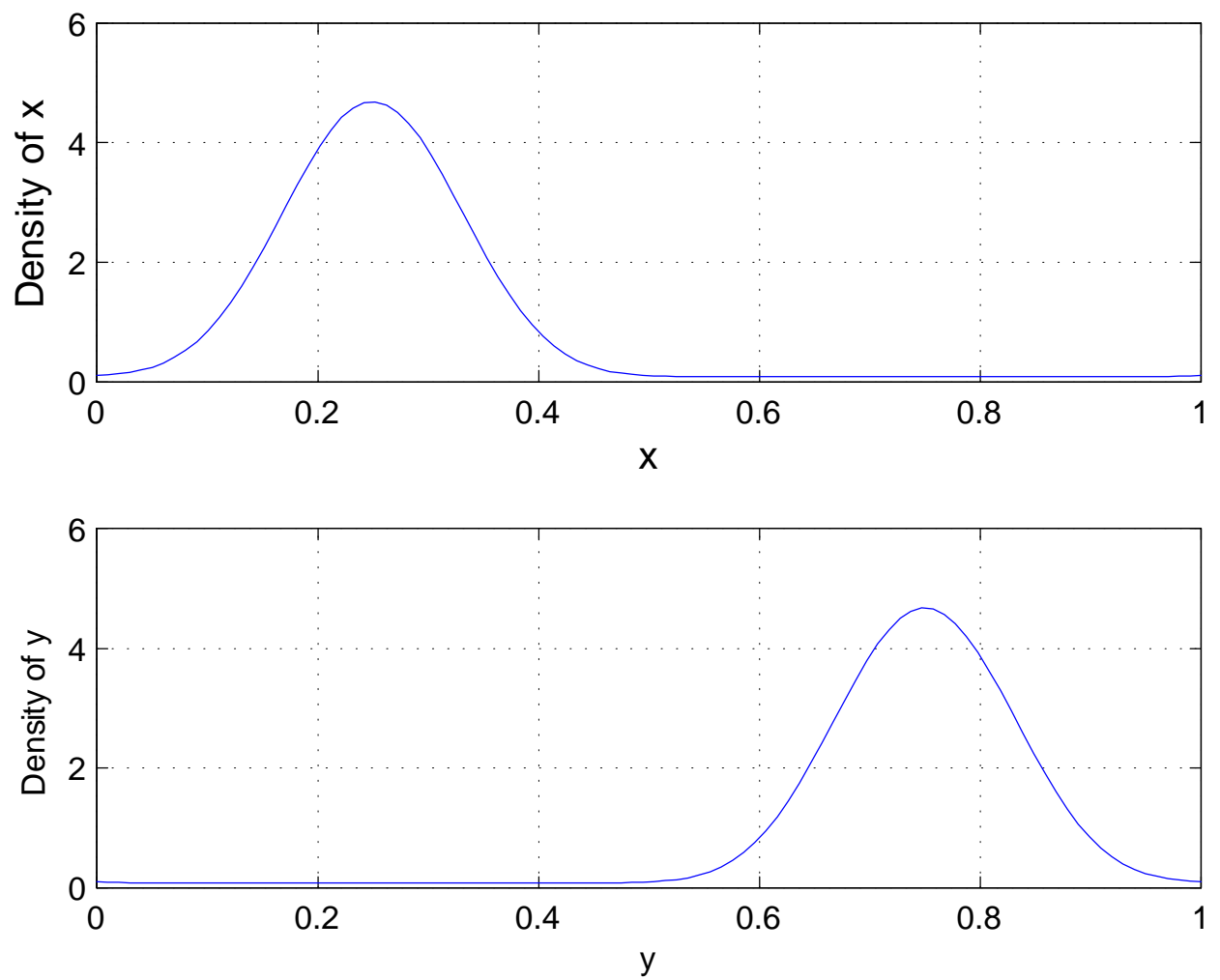
Figure 2: Numerical support of optimal assignment in Example 4.17

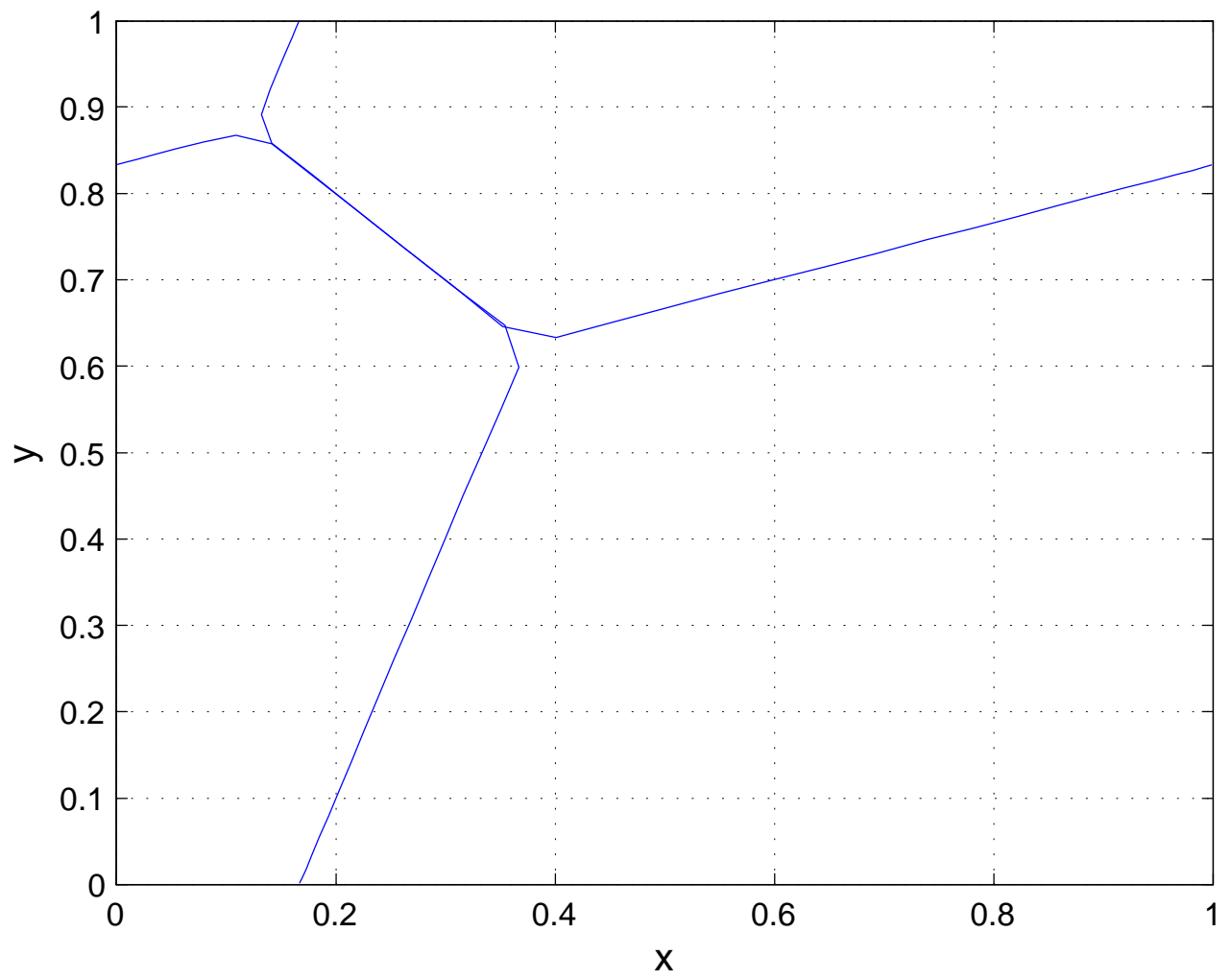


Figure 3: Indirect utilities of students and schools computed numerically
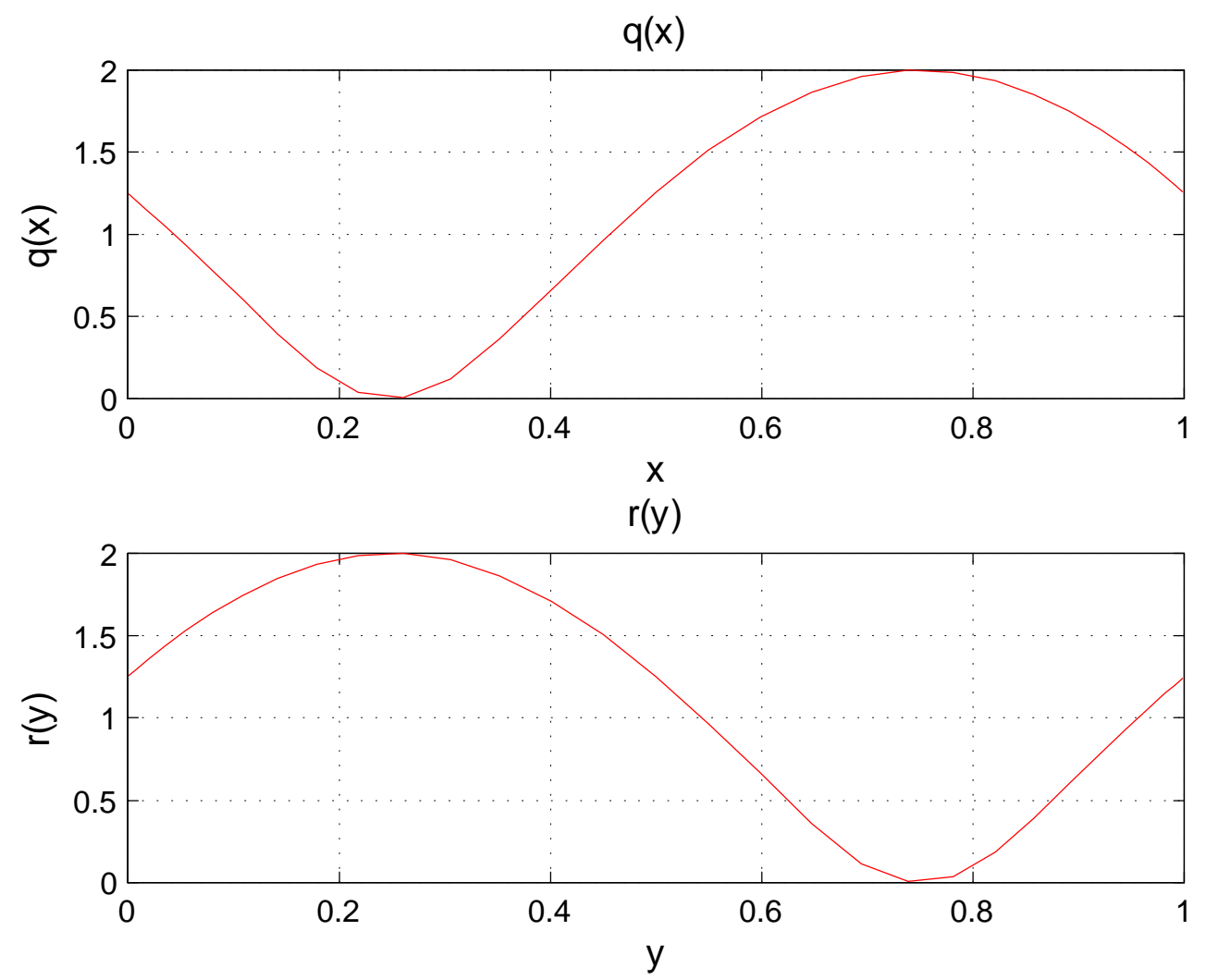